\title{
Four classes of pattern-avoiding permutations under one roof: generating trees with two labels
}

\author{
Mireille Bousquet-MÉlou* \\ CNRS, LaBRI, Université Bordeaux 1 \\ 351 cours de la Libération \\ 33405 Talence Cedex, France \\ mireille.bousquet@labri.fr
}

Submitted: Sep 11, 2003; Accepted: Oct 13, 2003; Published: Nov 7, 2003

MR Subject Classifications: 05A15, 05A10

\begin{abstract}
Many families of pattern-avoiding permutations can be described by a generating tree in which each node carries one integer label, computed recursively via a rewriting rule. A typical example is that of 123-avoiding permutations. The rewriting rule automatically gives a functional equation satisfied by the bivariate generating function that counts the permutations by their length and the label of the corresponding node of the tree. These equations are now well understood, and their solutions are always algebraic series.

Several other families of permutations can be described by a generating tree in which each node carries two integer labels. To these trees correspond other functional equations, defining 3 -variate generating functions. We propose an approach to solving such equations. We thus recover and refine, in a unified way, some results on Baxter permutations, 1234-avoiding permutations, 2143-avoiding (or: vexillary) involutions and 54321-avoiding involutions.

All the generating functions we obtain are D-finite, and, more precisely, are diagonals of algebraic series. Vexillary involutions are exceptionally simple: they are counted by Motzkin numbers, and thus have an algebraic generating function.

In passing, we exhibit an interesting link between Baxter permutations and the Tutte polynomial of planar maps.
\end{abstract}

*Partially supported by the European Community IHRP Program, within the Research Training Network "Algebraic Combinatorics in Europe", grant HPRN-CT-2001-00272. 

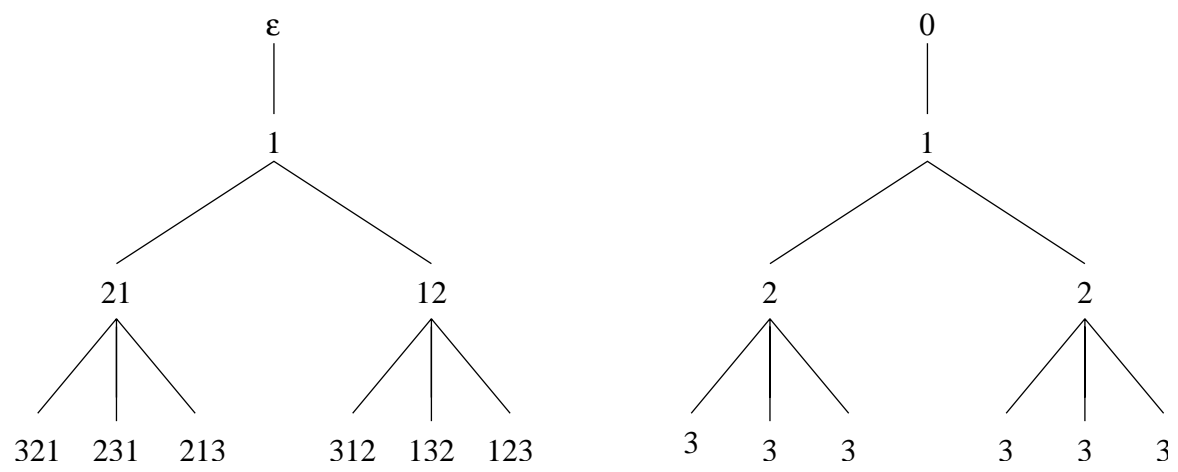

Figure 1: (a) The generating tree of permutations. (b) Nodes labeled by the length of the permutations.

\section{Introduction}

\subsection{Pattern-avoiding permutations and generating trees}

Let $\sigma=\sigma_{1} \sigma_{2} \cdots \sigma_{n}$ be a permutation of length $n$. Let $\tau=\tau_{1} \cdots \tau_{k}$ be a permutation of length $k \leq n$. We say that $\sigma$ contains the pattern $\tau$ if there exist $i_{1}<i_{2}<\cdots<i_{k}$ such that the standardization of the word $\sigma_{i_{1}} \cdots \sigma_{i_{k}}$ gives $\tau_{1} \cdots \tau_{k}$. In other words, $\sigma_{i_{j}}<\sigma_{i_{\ell}}$ if and only if $\tau_{j}<\tau_{\ell}$. Otherwise, we say that $\sigma$ avoids $\tau$. We denote by $\mathcal{S}(\tau)$ the set of $\tau$-avoiding permutations.

The enumeration of permutations constrained to avoid certain patterns has received a lot of attention in the last few years; see for instance $[1,5,12,15,21,23,32,33]$. The use of generating trees, which has been systematized by West [37, 38], is natural in this context. The generating tree $T$ of unrestricted permutations is shown in Figure 1: its root is indexed by the empty permutation, and a node indexed by a permutation $\sigma$ of length $n$ has $n+1$ children, respectively indexed by the $n+1$ permutations that can be obtained by inserting the letter $(n+1)$ in the word $\sigma_{1} \sigma_{2} \cdots \sigma_{n}$. Clearly, this tree is isomorphic to a simpler tree, in which the root is labelled 0 , and a node labelled $n$ has $n+1$ children, each labelled by $(n+1)$. The latter tree can be described succintly by the following rewriting rule:

$$
(n) \sim(n+1)^{n+1} .
$$

A similar procedure, consisting of inserting a new cycle, exists for involutions; it will be described and used in Sections 4 and 5.

These trees are well-suited to the study of permutations avoiding patterns, because all ancestors of a permutation avoiding a pattern $\tau$ also avoid $\tau$. Consequently, permutations avoiding $\tau$ form a subtree $T_{\tau}$ of $T$. In some cases, $T_{\tau}$ can be shown to be isomorphic to a tree in which the nodes carry a simple label that can be computed recursively using a rewriting rule. 

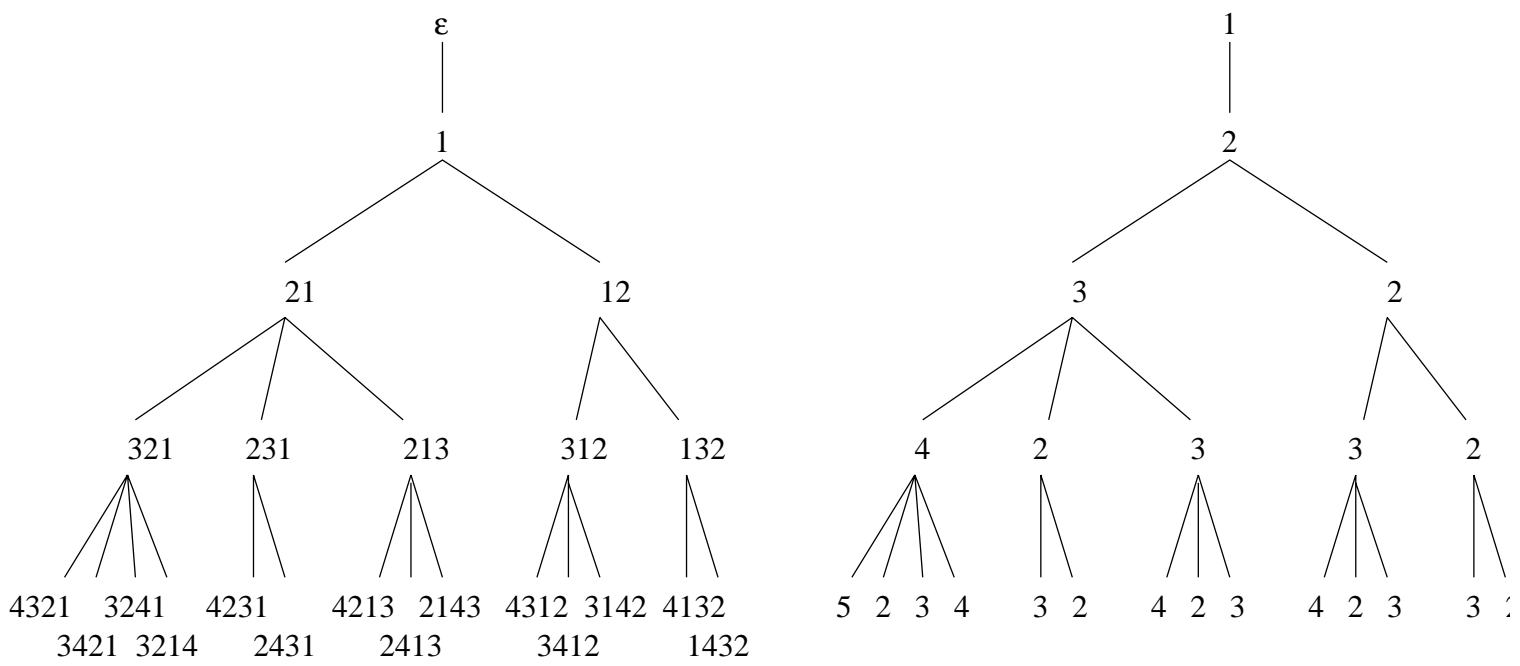

Figure 2: (a) The generating tree of 123-avoiding permutations. (b) Nodes labeled by the position of the first rise.

\subsection{Generating trees with one label}

Consider, for instance, permutations avoiding 123 . In the tree $T_{123}$, replace each permutation by the position of its first rise (which is taken in the interval $[2, n+1]$ for a permutation of length $n$ ); see Figure 2. The resulting tree can be described by the following rewriting rule:

$$
(p) \sim(p+1)(2)(3) \cdots(p) .
$$

Let $G(t ; u) \equiv G(u)$ be the associated generating function:

$$
G(t ; u)=\sum_{\sigma \in \mathcal{S}(123)} t^{\ell(\sigma)} u^{p(\sigma)}:=\sum_{p \geq 1} G_{p}(t) u^{p}
$$

where $\ell(\sigma)$ denotes the length of $\sigma$, and $p(\sigma)$ the position of its first rise. Alternatively, this series counts the nodes of the tree by their height and their label (the root being at height 0 ). Underlying the rule (1) is the following functional equation:

$$
\begin{aligned}
G(u) & =u+t \sum_{p \geq 1} G_{p}(t)\left(u^{2}+\cdots+u^{p}+u^{p+1}\right) \\
& =u+t u^{2} \frac{G(u)-G(1)}{u-1} .
\end{aligned}
$$

This type of equation can be solved systematically using the kernel method [2]. In this case, one recovers the well-known enumeration of 123-avoiding permutations by Catalan numbers:

$$
G(t ; 1)=\frac{1-\sqrt{1-4 t}}{2 t}=\sum_{n \geq 0} \frac{1}{n+1}\left(\begin{array}{c}
2 n \\
n
\end{array}\right) t^{n} .
$$


In a fairly large number of cases, the permutations avoiding a given set of patterns can be described by a generating tree in which the nodes carry one integer label [17, 19, 20, 24, 38]. The corresponding functional equations can be solved routinely by the kernel method, always yielding algebraic generating functions ${ }^{1}$. A systematic approach to these equations is presented in [2].

\subsection{Generating trees with two labels}

On the contrary, trees defined by a rewriting rule with two labels have never been submitted to a frontal attack. Still, they occur naturally in the enumeration of pattern avoiding permutations.

A striking example is that of vexillary (2143-avoiding) involutions. In 1995, Guibert conjectured they were counted by Motzkin numbers [19]. This apparently simple conjecture resisted for several years, until in 2001, Guibert, Pergola and Pinzani gave a rather complicated, recursive bijective proof [21]. However, in 1995 already, Guibert had given a simple description, with two labels, of the generating tree of these involutions. The associated rewriting rule could readily be translated into the following functional equation, defining a 3 -variate generating function $G(t ; u, v) \equiv G(u, v)$ :

$$
\left(1+\frac{t^{2} u^{2} v}{1-u}+\frac{t^{2} v}{1-v}\right) G(u, v)=\frac{u v(1-t)}{1-u v t}+t\left(1+\frac{t v}{1-v}\right) G(u v, 1)+\frac{t^{2} u^{2} v}{1-u} G(1, v) .
$$

The variable $t$ takes into account the length of the involutions, while $u$ and $v$ correspond to two additional statistics that will be described in Section 4. This equation somehow solves the problem of counting vexillary involutions, and it is really vexing not to be able to derive from it that $G(1,1)$ is the generating function of Motzkin numbers:

$$
G(1,1)=\frac{1-t-\sqrt{(1+t)(1-3 t)}}{2 t^{2}}
$$

The aim of this paper is to remedy this frustration by solving (2) and three other equations of the same type. Each of them defines the generating function of a class of pattern-avoiding permutations that can be described by a bi-labelled generating tree: we thus recover and refine, in a unified way, some results on Baxter permutations, 1234avoiding permutations and 54321-avoiding involutions.

Let us replace $u$ by $u / v$ in $(2)$, and denote $H(u, v)=G(u / v, v)$. The functional equation becomes:

$$
\left(1+\frac{t^{2} u^{2}}{v-u}+\frac{t^{2} v}{1-v}\right) H(u, v)=\frac{u(1-t)}{1-u t}+t\left(1+\frac{t v}{1-v}\right) H(u, 1)+\frac{t^{2} u^{2}}{v-u} H(v, v) .
$$

\footnotetext{
${ }^{1} \mathrm{~A}$ series $F(t)$ is algebraic if it satisfies a polynomial equation $P(t, F(t))=0$.
} 
More generally, all the equations we are going to study are linear combinations of

- one main 3 -variate series $H(t ; u, v)$,

- a number of series that do not depend on $u$ and $v$ simultaneously.

The coefficients of this linear combination are polynomials in $t, u, v$. The coefficient of $H(t ; u, v)$ is called the kernel of the equation. Following Zeilberger [39], we call these equations linear equations with two catalytic variables $u$ and $v$.

Linear equations with two catalytic variables do not only occur in the enumeration of pattern-avoiding permutations. It happens quite often that the objects one wishes to count admit a recursive description that forces us to keep track of certain secondary statistics (in addition to the size of the objects). If there are two secondary statistics, then the enumeration of the objects is likely to be governed by an equation with two catalytic variables. In particular, planar walks confined in a quadrant provide a wide class of such equations (in this case, the secondary statistics are the coordinates of the endpoint). It was recently shown that, depending on the steps the walk is allowed to take, the associated generating function can be algebraic, D-finite but transcendental ${ }^{2}$, or even non-D-finite $[7,8,10]$. This is in sharp contrast to the case of a single catalytic variable, which invariably yields algebraic solutions.

The four equations solved in this paper have D-finite solutions. More precisely, the solutions are expressed as diagonals of algebraic series (precise definitions will be given below). Our approach to solving these equations uses two steps: the first step is again the kernel method, or rather an obstinate variation of it that was inspired to us by the book [14]. This step can be applied systematically to any linear equation with two catalytic variables. It yields a system of equations that are nicer than the original one, because they relate series involving only one catalytic variable. However, they are also worse than the original equation, because they involve certain algebraic substitutions. The second step is more mysterious, and seems to depend strongly on the kernel of the original equation. The idea is to form "nice" linear combinations of the equations provided by the first step, from which one can easily extract the positive part. Giving more details here would require us to be more technical. The four examples presented below provide ample illustration of this second step. The first example - Baxter permutations - is especially striking: the only calculation our solution requires is an application of the Lagrange inversion formula.

Let us mention that this two-step approach was used already in $[7,8]$ to count lattice walks confined in a quadrant. Then, we tried it successfully on vexillary involutions. Then, we tried it on all bi-labelled generating trees we could find in the world of patternavoiding permutations - and, to our surprise, the approach kept working, as is reported in this paper. A more recent example is provided by osculating walks [6]. Needless to say, we would be interested in trying this method on other (combinatorially founded) trees with two labels: all examples are welcome!

\footnotetext{
${ }^{2} \mathrm{~A}$ series $F(t)$ is D-finite if it satisfies a linear differential equation with polynomial coefficients.
} 


\subsection{Definitions and notations}

Let us conclude this section by giving some definitions and notations on permutations and formal power series. The group of permutations of length $n$ will be denoted by $\mathcal{S}_{n}$. We shall use both the word representation of a permutation, $\sigma=\sigma_{1} \sigma_{2} \cdots \sigma_{n}$, and its factorization into disjoint cycles.

Given a ring $\mathbb{L}$ and $k$ indeterminates $x_{1}, \ldots, x_{k}$, we denote by $\mathbb{L}\left[x_{1}, \ldots, x_{k}\right]$ the ring of polynomials in $x_{1}, \ldots, x_{k}$ with coefficients in $\mathbb{L}$. We denote by $\mathbb{L}\left[\left[x_{1}, \ldots, x_{k}\right]\right]$ the ring of formal power series in the $x_{i}$, that is, of formal sums

$$
\sum_{n_{1} \geq 0, \ldots, n_{k} \geq 0} a\left(n_{1}, \ldots, n_{k}\right) x_{1}^{n_{1}} \cdots x_{k}^{n_{k}}
$$

where $a\left(n_{1}, \ldots, n_{k}\right) \in \mathbb{L}$. A Laurent polynomial in the $x_{i}$ is a polynomial in the $x_{i}$ and the $\bar{x}_{i}=1 / x_{i}$. A Laurent series in the $x_{i}$ is a series of the form (3) in which the summation runs over $n_{i} \geq m_{i}$ for all $i$, with $m_{i}$ in $\mathbb{Z}$.

For $F \in \mathbb{L}[[t]]$, we denote by $\left[t^{n}\right] F$ the coefficient of $t^{n}$ in $F(t)$. Similarly, if $F$ is a formal series in $t$ whose coefficients are Laurent series in $x$, we denote by $\left[x^{i} t^{n}\right] F$ the coefficient of $x^{i}$ in $\left[t^{n}\right] F$. We denote by $F^{>}$the positive part of $F$ in $x$, that is,

$$
F=\sum_{n \geq 0} t^{n} \sum_{i \in \mathbb{Z}} f(n, i) x^{i} \Longrightarrow F^{>}=\sum_{n \geq 0} t^{n} \sum_{i>0} f(n, i) x^{i}
$$

We define accordingly the nonnegative part of $F$ in $x$, and denote it by $F^{\geq}$.

Assume, from now on, that $\mathbb{L}$ is a field. We denote by $\mathbb{L}\left(x_{1}, \ldots, x_{k}\right)$ the field of rational functions in $x_{1}, \ldots, x_{k}$ with coefficients in $\mathbb{L}$. A series $F$ in $\mathbb{L}\left[\left[x_{1}, \ldots, x_{k}\right]\right]$ is rational if there exist polynomials $P$ and $Q$ in $\mathbb{L}\left[x_{1}, \ldots, x_{k}\right]$, with $Q \neq 0$, such that $Q F=P$. It is algebraic if there exists a non-trivial polynomial $P$ with coefficients in $\mathbb{L}$ such that $P\left(F, x_{1}, \ldots, x_{k}\right)=0$. It is $D$-finite if the partial derivatives of $F$ span a finite dimensional vector space over the field $\mathbb{L}\left(x_{1}, \ldots, x_{k}\right)$; see [34] for the one-variable case, and [26, 27] otherwise. In other words, for $1 \leq i \leq k$, the series $F$ satisfies a non-trivial partial differential equation of the form

$$
\sum_{\ell=0}^{d_{i}} P_{\ell, i} \frac{\partial^{\ell} F}{\partial x_{i}^{\ell}}=0
$$

where $P_{\ell, i}$ is a polynomial in the $x_{j}$. Any algebraic series is D-finite. The specializations of a D-finite series (obtained by giving values from $\mathbb{L}$ to some of the variables) are D-finite, if well-defined. Finally, if $F$ is D-finite, then any diagonal of $F$ is also D-finite [26] (the diagonal of $F$ in $x_{1}$ and $x_{2}$ is obtained by keeping only those monomials for which the exponents of $x_{1}$ and $x_{2}$ are equal). We shall use the following consequence of this result: if $F(t, x) \in \mathbb{L}[x, \bar{x}][[t]]$ is algebraic, then the positive part of $F$ in $x$ is D-finite, as well as the coefficient of $x^{i}$ in this series, for all $i$. 


\section{Baxter permutations}

A permutation $\sigma=\sigma_{1} \cdots \sigma_{n}$ is said to be a Baxter permutation if, for any $i \in\{1, \ldots, n-$ $1\}$, the word $\sigma$ can be written either as

$$
\sigma=\pi i \pi_{-} \pi_{+}(i+1) \pi^{\prime}
$$

or as

$$
\sigma=\pi(i+1) \pi_{+} \pi_{-} i \pi^{\prime}
$$

where all letters occurring in $\pi_{+}$(resp. $\pi_{-}$) are larger (resp. smaller) than $i$. For instance, all permutations of length 4 are Baxter permutations except 2413 and 3142 (check $i=2$ ). Our aim is to recover and refine the following result.

Theorem 1 The number of Baxter permutations of $\mathcal{S}_{n}$ is

$$
\frac{2}{n(n+1)^{2}} \sum_{k=1}^{n}\left(\begin{array}{c}
n+1 \\
k-1
\end{array}\right)\left(\begin{array}{c}
n+1 \\
k
\end{array}\right)\left(\begin{array}{l}
n+1 \\
k+1
\end{array}\right) .
$$

This is sequence A001181 in the on-line Encyclopedia of Integer Sequences [31]. It starts with $1,2,6,22,92,422 \ldots$ The first proof of this result is due to Chung, Graham, Hoggatt and Kleiman [11]. Other proofs were given by Mallows [28], Viennot [36], Dulucq and Guibert [13]. Bexter permutations can be described in terms of generalized forbidden patterns [17].

\subsection{Recursive construction of Baxter permutations}

Let $\sigma$ be a Baxter permutation of length $n$, and let $\tau$ be obtained by deleting the letter $n$ from $\sigma$. Then $\tau$ is a Baxter permutation as well. Conversely, let us try to contruct a Baxter permutation of length $n+1$ by inserting the letter $(n+1)$ in $\sigma$. It is not very hard to see that $(n+1)$ has to be inserted:

- either just before a left-to-right maximum of $\sigma$, or

- just after a right-to-left maximum of $\sigma$.

We are thus led to introduce two additional statistics, namely the number of left-to-right maxima and the number of right-to-left maxima of $\sigma$, which we call loosely the parameters of $\sigma$.

Lemma 2 ([17]) Let $\sigma$ be a Baxter permutation of length $n \geq 1$, of parameters $(p, q)$. Exactly $p+q$ Baxter permutations can be obtained by inserting $(n+1)$ in $\sigma$, and their parameters are respectively:

$$
\begin{aligned}
& (1, q+1),(2, q+1), \ldots,(p, q+1), \\
& (p+1, q),(p+1, q-1), \ldots,(p+1,1) .
\end{aligned}
$$

The order in which the parameters are listed corresponds to the insertion positions visited from left to right. 
For $p, q \geq 1$, let $G_{p, q}(t) \equiv G_{p, q}$ denote the length generating function of Baxter permutations having parameters $p$ and $q$. Let

$$
G(t ; u, v) \equiv G(u, v)=\sum_{p, q \geq 1} G_{p, q} u^{p} v^{q} .
$$

The above lemma gives

$$
\begin{aligned}
G(u, v) & =t u v+t \sum_{p, q \geq 1} G_{p, q}\left(\left(u+u^{2}+\cdots+u^{p}\right) v^{q+1}+u^{p+1}\left(v^{q}+v^{q-1}+\cdots+v\right)\right), \\
& =t u v+t \sum_{p, q \geq 1} G_{p, q}\left(\frac{u-u^{p+1}}{1-u} v^{q+1}+u^{p+1} \frac{v-v^{q+1}}{1-v}\right) .
\end{aligned}
$$

We thus obtain the following result.

Corollary 3 Let $G(t ; u, v) \equiv G(u, v)$ denote the generating function of non-empty Baxter permutations, counted by their length (variable $t$ ) and parameters (variables $u$ and $v$ ). Then

$$
\left(1+\frac{t u v}{1-u}+\frac{t u v}{1-v}\right) G(u, v)=t u v+\frac{t u v}{1-v} G(u, 1)+\frac{t u v}{1-u} G(1, v) .
$$

Note that $G(u, v)$ is symmetric in $u$ and $v$. In particular, $G(u, 1)=G(1, u)$. It will be convenient to set $u=1+x$ and $v=1+y$. The equation becomes

$$
\frac{x y-t(1+x)(1+y)(x+y)}{t(1+x)(1+y)} G(1+x, 1+y)=x y-R(x)-R(y)
$$

with $R(x)=x G(1+x, 1)$.

\subsection{Solution of the functional equation for Baxter permutations}

Theorem 4 Let $Z(t ; x) \equiv Z$ be the unique formal power series in $t$ such that

$$
Z=t(1+x+Z)(1+\bar{x}+Z) .
$$

This series has coefficients in $\mathbb{Q}[x, \bar{x}]$, with $\bar{x}=1 / x$. The series $G(t ; u, 1)$ that counts Baxter permutations by their length and number of left-to-right maxima satisfies:

$$
x G(t ; 1+x, 1)=\left(1+(x+\bar{x}) Z-\frac{Z}{t(1+x)(1+\bar{x})}\right)^{>} .
$$

This shows that the series $G(t ; u, 1)$ is D-finite, and Corollary 3 then implies that $G(t ; u, v)$ is D-finite too. The Lagrange inversion formula gives: 
Corollary 5 The series $G(t ; u, 1)$ admits the following expansions:

$$
G(t ; 1+x, 1)=\sum_{n \geq 1} t^{n} \sum_{i=0}^{n} \frac{x^{i}(i+1)}{n(n+1)^{2}(n+2)} \sum_{k=i}^{n}(2 k+n i)\left(\begin{array}{c}
n+2 \\
k-i
\end{array}\right)\left(\begin{array}{c}
n+1 \\
k
\end{array}\right)\left(\begin{array}{c}
n+1 \\
k+1
\end{array}\right)
$$

and

$$
G(t ; u, 1)=\sum_{n \geq 1} t^{n}\left(u^{n}+\sum_{i=1}^{n-1} \frac{u^{i} i(i+1)}{n(n+1)^{2}} \sum_{k=1}^{n-i}\left(\begin{array}{c}
n+1 \\
k
\end{array}\right)\left(\begin{array}{c}
n+1 \\
k+1
\end{array}\right)\left(\begin{array}{c}
n-i-1 \\
k-1
\end{array}\right)\right) .
$$

Note that the case $x=0$ of (5) is exactly Theorem 1 .

Proof of Theorem 4. Let us consider Eq. (4). We call the coefficient of $G(1+x, 1+y)$ (or, more precisely, its numerator) the kernel $K(x, y)$ of the equation:

$$
K(x, y)=x y-t(1+x)(1+y)(x+y) .
$$

We are going to apply to Eq. (4) the so-called kernel method. It has been around at least since the 70 's, and is currently the subject of a certain revival (see the references in $[2,3,9])$. It consists in coupling the variables $x$ and $y$ so as to cancel the kernel. This should give the "missing" information about the series $R(x)$.

As a polynomial in $y$, the kernel has two roots:

$$
\begin{aligned}
Y_{0}(x) & =\frac{1-t(1+x)(1+\bar{x})-\sqrt{1-2 t(1+x)(1+\bar{x})-t^{2}\left(1-x^{2}\right)\left(1-\bar{x}^{2}\right)}}{2 t(1+\bar{x})} \\
& =(1+x) t+(1+x)^{2}(1+\bar{x}) t^{2}+O\left(t^{3}\right), \\
Y_{1}(x) & =\frac{1-t(1+x)(1+\bar{x})+\sqrt{1-2 t(1+x)(1+\bar{x})-t^{2}\left(1-x^{2}\right)\left(1-\bar{x}^{2}\right)}}{2 t(1+\bar{x})} \\
& =\frac{x}{1+x} \frac{1}{t}-(1+x)-(1+x) t+O\left(t^{2}\right) .
\end{aligned}
$$

Observe that $Y_{0} Y_{1}=x$.

Only the first root can be substituted for $y$ in (4) (the term $G\left(1+x, 1+Y_{1}\right.$ ) is not a well-defined power series in $t$ ). We thus obtain a functional equation for $R(x)$ :

$$
R(x)+R\left(Y_{0}\right)=x Y_{0}
$$

It can be shown that this equation uniquely defines $R(x)$ as a formal power series in $t$ with coefficients in $x \mathbb{Q}[x]$. Equation (7) is the standard result of the kernel method.

Still, as in $[7,8]$, we want to apply here the obstinate kernel method. That is, we shall not content ourselves with (7), but we shall go on producing pairs $(X, Y)$ that cancel the kernel and use the information they provide on the series $R(x)$. This obstination was inspired by the book [14] by Fayolle, Iasnogorodski and Malyshev, and more precisely by Section 2.4 of this book, where one possible way to obtain such pairs is described (even though the analytic context is different). We give here an alternative construction. 
Let $(X, Y) \neq(0,0)$ be a pair of Laurent series in $t$ with coefficients in a field $\mathbb{K}$ such that $K(X, Y)=0$. We define $\Phi(X, Y)=\left(X^{\prime}, Y\right)$, where $X^{\prime}$ is the other solution of $K(x, Y)=0$, seen as a polynomial in $x$ (remember that $K$ has degree 2 in $x$ ). Similarly, we define $\Psi(X, Y)=\left(X, Y^{\prime}\right)$, where $Y^{\prime}$ is the other solution of $K(X, y)=0$. Note that $\Phi$ and $\Psi$ are involutions. Moreover, with the kernel given by (6), one has $Y^{\prime}=X / Y$ and $X^{\prime}=Y / X$. Let us examine the action of $\Phi$ and $\Psi$ on the pair $\left(x, Y_{0}\right)$ : we obtain an orbit of cardinality 6 (Figure 3). A geometric description of this orbit is provided in Figure 4.

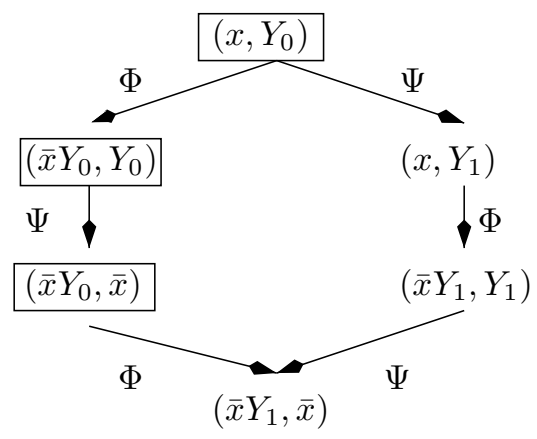

Figure 3: The orbit of $\left(x, Y_{0}\right)$ under the action of $\Phi$ and $\Psi$.

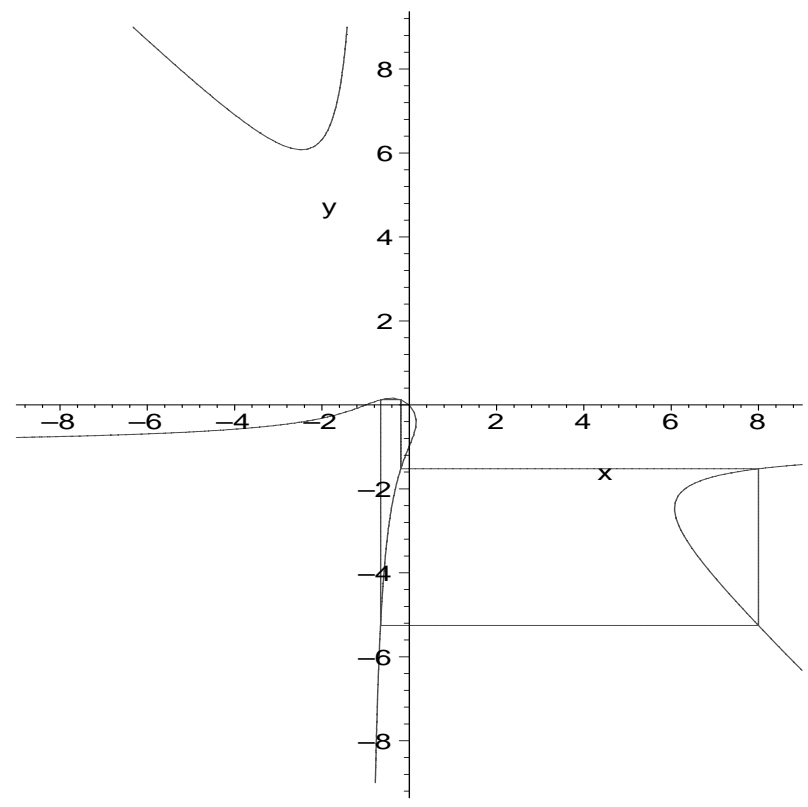

Figure 4: The real part of the curve $K(t ; x, y)=0$ for $t=0.4$. Applying the transformations $\Phi$ and $\Psi$ corresponds to moving from one branch of the curve to another, along the $x$ - and $y$-axes.

The 6 pairs of power series given in Figure 3 cancel the kernel, and we have framed the ones that can be legally substituted for $(x, y)$ in the main functional equation (4). We 
thus obtain three equations for the unknown series $R(x)$ :

$$
\left\{\begin{array}{l}
R(x)+R\left(Y_{0}\right)=x Y_{0} \\
R\left(\bar{x} Y_{0}\right)+R\left(Y_{0}\right)=\bar{x} Y_{0}^{2} \\
R\left(\bar{x} Y_{0}\right)+R(\bar{x})=\bar{x}^{2} Y_{0}
\end{array}\right.
$$

By combining these three equations, we obtain a relation between $R(x)$ and $R(\bar{x})$ :

$$
R(x)+R(\bar{x})=\bar{x}^{2} Y_{0}\left(1+x^{3}-x Y_{0}\right) .
$$

But $R(x)=x G(1+x, 1)$ is a formal power series in $t$ with coefficients in $x \mathbb{Q}[x]$, while $R(\bar{x})$ is a formal power series in $t$ with coefficients in $\bar{x} \mathbb{Q}[\bar{x}]$. Hence the positive part in $x$ of the right-hand side is exactly $R(x)$. What remains is to observe that $Y_{0}$ is related to the series $Z$ defined in Theorem 4 by $Y_{0}=Z /(1+\bar{x})$, and to express the right-hand side of (8) as a polynomial of degree 1 in $Z$ to complete the proof of Theorem 4.

Remark. It is certainly more natural to start with the original variables $u$ and $v$. The same method provides a relation between $G(u, 1)$ and $G(u /(u-1), 1)$, and at this point, the change of variables $u=1+x, v=1+y$ becomes natural.

Proof of Corollary 5. The Lagrange inversion formula gives, for $n \geq 1$,

$$
\left[t^{n}\right] Z=\frac{1}{n} \sum_{k=1}^{n}\left(\begin{array}{c}
n \\
k-1
\end{array}\right)\left(\begin{array}{l}
n \\
k
\end{array}\right)(1+x)^{k}(1+\bar{x})^{n+1-k} .
$$

Let us denote

$$
C(t ; x) \equiv C(x)=1+(x+\bar{x}) Z-\frac{Z}{t(1+x)(1+\bar{x})} .
$$

Recall that $x G(1+x, 1)=C(t ; x)^{>}$. Then for $n \geq 1$,

$$
\begin{aligned}
{\left[t^{n}\right] C(x)=\frac{x+\bar{x}}{n} \sum_{k=1}^{n}\left(\begin{array}{c}
n \\
k-1
\end{array}\right)\left(\begin{array}{l}
n \\
k
\end{array}\right) } & (1+x)^{k}(1+\bar{x})^{n+1-k} \\
& -\frac{1}{n+1} \sum_{k=1}^{n+1}\left(\begin{array}{c}
n+1 \\
k-1
\end{array}\right)\left(\begin{array}{c}
n+1 \\
k
\end{array}\right)(1+x)^{k-1}(1+\bar{x})^{n+1-k} .
\end{aligned}
$$

Given that

$$
\left[x^{j}\right](1+x)^{k}(1+\bar{x})^{\ell}=\left(\begin{array}{c}
k+\ell \\
k-j
\end{array}\right)
$$

we find

$$
\begin{aligned}
& {\left[x^{i+1} t^{n}\right] C(t ; x)=\frac{1}{n} \sum_{k=1}^{n}\left(\begin{array}{c}
n \\
k-1
\end{array}\right)\left(\begin{array}{l}
n \\
k
\end{array}\right)\left(\begin{array}{l}
n+1 \\
k-i
\end{array}\right)} \\
& \quad+\frac{1}{n} \sum_{k=1}^{n}\left(\begin{array}{c}
n \\
k-1
\end{array}\right)\left(\begin{array}{l}
n \\
k
\end{array}\right)\left(\begin{array}{c}
n+1 \\
k-i-2
\end{array}\right)-\frac{1}{n+1} \sum_{k=1}^{n+1}\left(\begin{array}{c}
n+1 \\
k-1
\end{array}\right)\left(\begin{array}{c}
n+1 \\
k
\end{array}\right)\left(\begin{array}{c}
n \\
k-i-2
\end{array}\right) .
\end{aligned}
$$


Upon summing the $k$ th term in the first summation and the $(k+1)$ th terms of the second and third summation, one obtains

$$
\left[x^{i+1} t^{n}\right] C(t ; x)=\sum_{k=i}^{n} \frac{(2 k+n i)(i+1)}{n(n+1)^{2}(n+2)}\left(\begin{array}{c}
n+2 \\
k-i
\end{array}\right)\left(\begin{array}{c}
n+1 \\
k
\end{array}\right)\left(\begin{array}{c}
n+1 \\
k+1
\end{array}\right) .
$$

The announced expansion of $G(1+x, 1)$ now follows from $x G(1+x, 1)=C(t ; x)^{>}$. In order to obtain the expansion of $G(u, 1)$ in $u$, we use the following lemma.

Lemma 6 Let $D(t ; x) \equiv D(x)$ be a formal power series in $t$ with coefficients in $\mathbb{Q}[x, \bar{x}]$. Let $S(1+x)$ be defined as the nonnegative part (in $x$ ) of $D(x)$. Let us consider $D(\bar{v}-1)$ as a series in $t$ whose coefficients are Laurent series in $v$. Then $S(\bar{v})$ is the nonpositive part of $D(\bar{v}-1)$ (in $v)$.

The proof is obvious by linearity, upon studying the case $D(x)=x^{n}$, for $n \in \mathbb{Z}$.

To complete the proof of Corollary 5, we now apply this lemma to $D(x)=C(x) / x$ and $S(1+x)=G(1+x, 1)$ : the series $G(\bar{v}, 1)$ is the nonpositive part of

$$
D(\bar{v}-1)=\frac{v}{1-v}+Z\left(1+\frac{v^{2}}{(1-v)^{2}}-\frac{v^{2}}{t}\right)
$$

with

$$
Z=t(\bar{v}+Z)\left(\frac{1}{1-v}+Z\right)
$$

The Lagrange inversion formula gives, for $n \geq 1$,

$$
\left[t^{n}\right] Z=\frac{1}{n} \sum_{k=0}^{n-1}\left(\begin{array}{l}
n \\
k
\end{array}\right)\left(\begin{array}{c}
n \\
k+1
\end{array}\right) \frac{\bar{v}^{n-k}}{(1-v)^{k+1}} .
$$

Given that

$$
\left[\bar{v}^{i}\right] \frac{\bar{v}^{a}}{(1-v)^{b+1}}=\left(\begin{array}{c}
a+b-i \\
b
\end{array}\right)
$$

one obtains, for $n \geq 1$ and $i \geq 1$,

$$
\begin{aligned}
{\left[t^{n} \bar{v}^{i}\right] G(\bar{v}, 1)=} & \frac{1}{n} \sum_{k=0}^{n-1}\left(\begin{array}{l}
n \\
k
\end{array}\right)\left(\begin{array}{c}
n \\
k+1
\end{array}\right)\left(\begin{array}{c}
n-i \\
k
\end{array}\right) \\
& +\frac{1}{n} \sum_{k=0}^{n-1}\left(\begin{array}{l}
n \\
k
\end{array}\right)\left(\begin{array}{c}
n \\
k+1
\end{array}\right)\left(\begin{array}{l}
n-i \\
k+2
\end{array}\right)-\frac{1}{n+1} \sum_{k=0}^{n-1}\left(\begin{array}{c}
n+1 \\
k
\end{array}\right)\left(\begin{array}{c}
n+1 \\
k+1
\end{array}\right)\left(\begin{array}{c}
n-i-1 \\
k
\end{array}\right) .
\end{aligned}
$$

The announced expansion of $G(u, 1)$ follows, upon grouping the $k$ th term of the first and third summation with the $(k-1)$ th term of the second summation. 


\subsection{The number of descents and the Tutte polynomial of planar maps}

A number of refinements of Theorem 1 and Corollary 5 exist. To our knowledge, the most refined version is due to Mallows [28], and takes into account the number of left-toright and right-to-left maxima, as well as the number of descents (see [13] for a bijective explanation of this result).

It is very easy to enrich the functional equation of Corollary 3 so as to take into account the number of descents: indeed, in the recursive construction of Baxter permutations, a new descent is created each time one performs an insertion before a left-to-right maximum. This gives the following refinement of Corollary 3:

$$
\left(1+\frac{t u v z}{1-u}+\frac{t u v}{1-v}\right) G(u, v)=t u v+\frac{t u v}{1-v} G(u, 1)+\frac{t u v z}{1-u} G(1, v),
$$

where $G(u, v) \equiv G(t, z ; u, v)$ now counts Baxter permutations by their length $(t)$, number of descents $(z)$, number of left-to-right and right-to-left maxima ( $u$ and $v$ ). The method of Section 2.2 applies verbatim, and provides the following counterparts of Theorem 1 and Corollary 5 .

Theorem 7 Let $Z(t, z ; x) \equiv Z$ be the unique formal power series in $t$ such that

$$
Z=t(1+x+z Z)(1+\bar{x}+Z) .
$$

This series has coefficients in $\mathbb{Q}[x, \bar{x}, z]$, with $\bar{x}=1 / x$. The series $G(t, z ; u, 1)$ that counts Baxter permutations by their length, number of descents and number of left-to-right maxima satisfies

$$
x G(t, z ; 1+x, 1)=\left(1+(x+z \bar{x}) Z-\frac{Z}{t(1+x)(1+\bar{x})}\right)^{>} .
$$

Corollary 8 The series $G(t, z ; u, 1)$ admits the following expansions:

$G(t, z ; 1+x, 1)=\sum_{n \geq 1} t^{n} \sum_{i=0}^{n} \frac{x^{i}(i+1)}{n(n+1)^{2}(n+2)} \sum_{k=i}^{n} z^{n-k}(2 k+n i)\left(\begin{array}{c}n+2 \\ k-i\end{array}\right)\left(\begin{array}{c}n+1 \\ k\end{array}\right)\left(\begin{array}{c}n+1 \\ k+1\end{array}\right)$,

and

$$
G(t, z ; u, 1)=\sum_{n \geq 1} t^{n}\left(u^{n}+\sum_{i=1}^{n-1} \frac{u^{i} i(i+1)}{n(n+1)^{2}} \sum_{k=1}^{n-i} z^{k}\left(\begin{array}{c}
n+1 \\
k
\end{array}\right)\left(\begin{array}{c}
n+1 \\
k+1
\end{array}\right)\left(\begin{array}{c}
n-i-1 \\
k-1
\end{array}\right)\right) .
$$

Let us define the series $T(s, t ; u, v) \equiv T(u, v)$ by $G(t, z ; u, v)=t u v T(t z, t ; u, v)$. The series $T(s, t ; u, v)$ is now a formal power series in $s$ and $t$ with coefficients in $\mathbb{Q}[u, v]$. It satisfies

$$
\left(1+\frac{s u v}{1-u}+\frac{t u v}{1-v}\right) T(u, v)=1+\frac{t u}{1-v} T(u, 1)+\frac{s v}{1-u} T(1, v) .
$$


Surprisingly, this equation also occurs in a recent study of the Tutte polynomial of planar maps [4, Eq. (5.2)]. Let us explain the combinatorial meaning of this observation. Let $\mathcal{M}_{m, n, i, j}$ be the set of rooted non-separable planar maps having $m+2$ faces, $n+2$ vertices, a root face of degree $i+1$ and a root vertex of degree $j+1$. For any map $M$, we denote by $\chi(M ; x, y)$ its Tutte polynomial. Then the coefficient of $x^{1} y^{0}$ in the polynomial

$$
\sum_{M \in \mathcal{M}_{m, n, i, j}} \chi(M ; x, y)
$$

is the number of Baxter permutations having $m$ descents, $n$ ascents, $i$ left-to-right maxima and $j$ right-to-left maxima. See Figure 5 for an illustration. The solution of (9) was guessed by the author of [4]. The approach presented in this paper allows us to derive it from the functional equation, without having to guess anything.

The connection between these two problems is all the more surprising that the author of [4] is (Rodney) Baxter, who did not recognize that the numbers he had guessed were related to (Glen) Baxter's permutations...

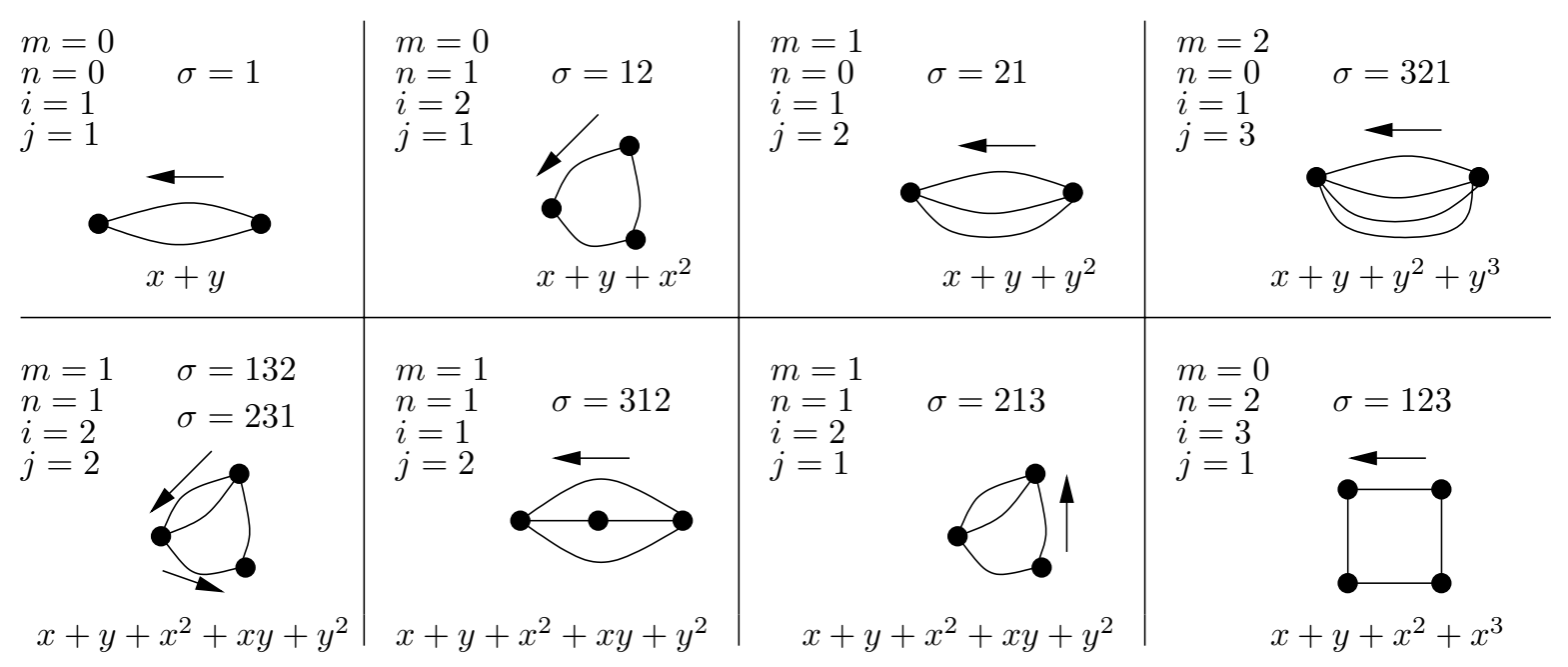

Figure 5: Non-separable planar maps, their Tutte polynomials, and the corresponding Baxter permutations. For the first map on the second row, two different rootings give the same value of $i$ and $j$.

\section{Permutations avoiding 1234}

We now focus on permutations containing no increasing subsequence of length 4 . We shall establish the following result.

Theorem 9 The number of 1234-avoiding permutations of $\mathcal{S}_{n}$ is

$$
\sum_{k=1}^{n}\left(\begin{array}{c}
2 k-2 \\
k-1
\end{array}\right)\left(\begin{array}{c}
n+2 \\
k
\end{array}\right)\left(\begin{array}{l}
n \\
k
\end{array}\right) \frac{2 n k-3 k^{2}+4 k-n}{n(n+1)(n+2)}
$$


This number admits the following simpler expression:

$$
\frac{1}{(n+1)^{2}(n+2)} \sum_{k=0}^{n}\left(\begin{array}{c}
2 k \\
k
\end{array}\right)\left(\begin{array}{c}
n+1 \\
k+1
\end{array}\right)\left(\begin{array}{l}
n+2 \\
k+1
\end{array}\right) \text {. }
$$

This is sequence A005802 in the on-line Encyclopedia of Integer Sequences [31]. It starts with 1, 2, 6, 23, 103, 513 . . By the Robinson-Schensted correspondence, these numbers also count pairs of standard Young tableaux of height at most 3 having the same shape [30]. A first expression of these numbers, reminiscent of the first formula above, was obtained by Gessel [16], using symmetric functions. A second proof, based on a correspondence with lattice walks, is presented by Gessel, Weinstein and Wilf in [15]. The first expression above is new, and the second, simpler one, appears in exercise 7.16 in [35]. Their equivalence is proved routinely using Zeilberger's algorithm [29].

\subsection{Recursive construction of 1234-avoiding permutations}

Let $\sigma$ be a 1234-avoiding permutation and let $\tau$ be obtained by deleting the letter $n$ from $\sigma$. Then $\tau$ avoids 1234 as well. Conversely, let us try to contruct a 1234-avoiding permutation of length $n+1$ by inserting the letter $(n+1)$ in $\sigma$. We must not insert $(n+1)$ to the right of an increasing subsequence of length 3 . This leads us to introduce two additional statistics, namely the position of the first rise and the position of the first 123-pattern. More precisely, let

$$
p= \begin{cases}\min \{k \geq 2: \sigma(k-1)<\sigma(k)\} & \text { if } \sigma \text { contains } 12 \\ n+1 & \text { otherwise }\end{cases}
$$

and

$$
q= \begin{cases}\min \{k \geq 3: \exists(i, j) \text { s.t. } i<j<k \text { and } \sigma(i)<\sigma(j)<\sigma(k)\} & \text { if } \sigma \text { contains 123 } \\ n+1 & \text { otherwise. }\end{cases}
$$

We call $p$ and $q$ the parameters of $\sigma$. The parameters of the empty permutation (of length $0)$ are $(1,1)$. Note that for any permutation, $p \leq q$.

Lemma 10 ([37]) Let $\sigma$ be a 1234-avoiding permutation of length $n \geq 0$, of parameters $(p, q)$. Exactly $q$ 1234-avoiding permutations can be obtained by inserting $(n+1)$ in $\sigma$, and their parameters are respectively:

$$
\begin{array}{ll}
(p+1, q+1),(2, q+1), \ldots,(p, q+1), & \\
(p, p+1),(p, p+2), \ldots,(p, q) & \text { for } p<q .
\end{array}
$$

Corollary 11 Let $G(t ; u, v) \equiv G(u, v)$ denote the generating function of 1234-avoiding permutations, counted by their length (variable $t$ ) and parameters (variables $u$ and $v$ ). Then

$$
\left(1+\frac{t u^{2} v}{1-u}+\frac{t v}{1-v}\right) G(u, v)=u v+\frac{t v}{1-v} G(u v, 1)+\frac{t u^{2} v}{1-u} G(1, v) .
$$


Remark. The rewriting rule of Lemma 10 also describes the insertion of $(n+1)$ in permutations avoiding 1243, as well as in permutations avoiding 2143 (with different definitions of the parameters $p$ and $q$ ) $[37,38]$. The latter permutations are also called vexillary. Permutations avoiding 1234 are also known to be in bijection with permutations avoiding $4123[32]$.

\subsection{Solution of the functional equation for 1234-avoiding permu- tations}

Theorem 12 Let $A(t ; x) \equiv A(x)$ be the following formal power series in $t$ :

$$
A(x)=\frac{\bar{x}^{2}(1-2 t \bar{x}-t-x+x t)}{2(1-t-t \bar{x})} \sqrt{\frac{1-t \bar{x}-5 t-4 x t}{1-t-t \bar{x}}} .
$$

This series has coefficients in $\mathbb{Q}[x, \bar{x}]$, with $\bar{x}=1 / x$. The series $G(t ; 1, v)$ that counts 1234-avoiding permutations by their length and position of the first 123-pattern satisfies

$$
G(t ; 1,1+x)=1+\left[x^{0}\right] A(x)+\frac{x}{t} A^{\geq}(x) .
$$

Proof. The term $G(u v, 1)$ in (10) suggests to introduce a new variable $w$ such that $u=w / v$. The series $G(u, v)$ becomes a series in $t$ with coefficients in $\mathbb{Q}[v, w]$, and the right-hand side of (10) now involves $G(w, 1)$ and $G(1, v)$. Then, we set $w=1+\bar{x}$ and $v=1+y$. We shall explain later how one is led to this change of variables. The equation becomes

$$
\begin{aligned}
-(x y(1-x y)-t(x+y & \left.\left.+3 x y-x^{2} y^{2}\right)\right) G\left(\frac{1+\bar{x}}{1+y}, 1+y\right) \\
= & -y(1+x)(1-x y)+t(1+y)(1-x y) R(\bar{x})+t(1+x)^{2} S(y)
\end{aligned}
$$

with $R(\bar{x})=x G(1+\bar{x}, 1)$ and $S(y)=y G(1,1+y)$. The kernel of this equation is now symmetric in $x$ and $y$, and has again two roots:

$$
\begin{aligned}
Y_{0}(x) & =\frac{1-t \bar{x}-3 t-\sqrt{(1-t-t \bar{x})(1-5 t-4 t x-t \bar{x})}}{2 x(1-t)} \\
& =t+(\bar{x}+3+x) t^{2}+O\left(t^{3}\right) \\
Y_{1}(x) & =\frac{1-t \bar{x}-3 t+\sqrt{(1-t-t \bar{x})(1-5 t-4 t x-t \bar{x})}}{2 x(1-t)} \\
& =\bar{x}-(1+\bar{x})^{2} t+O\left(t^{2}\right)
\end{aligned}
$$

Observe that the symmetric functions of the roots are polynomials in $\bar{x}$ :

$$
Y_{0}+Y_{1}=\frac{\bar{x}(1-t \bar{x}-3 t)}{1-t} \quad \text { and } \quad Y_{0} Y_{1}=\frac{t \bar{x}}{1-t}
$$

Consequently, the following lemma, which already played a crucial role in [7, 8], holds. 
Lemma 13 Let $F(t ; u, v) \equiv F(u, v)$ be a power series in $t$ with coefficients in $\mathbb{C}[u, v]$, such that $F(u, v)=F(v, u)$. Then the series $F\left(t ; Y_{0}, Y_{1}\right)$ is a power series in $t$ with polynomial coefficients in $\bar{x}$. Moreover, the constant term of this series, taken with respect to $\bar{x}$, is $F(t ; 0,0)$.

Proof. All symmetric polynomials in $Y_{0}$ and $Y_{1}$ are polynomials in $Y_{0}+Y_{1}$ and $Y_{0} Y_{1}$.

Starting from $\left(x, Y_{0}\right)$, we now construct the diagram of the pairs $(X, Y)$ that cancel the kernel, following the same rules as in Section 2. The symmetry of the kernel in $x$ and $y$, and the fact that $x Y_{0} Y_{1}=t /(1-t)$ make the calculation especially easy. The diagram, given in Figure 6, also shows which pairs can be legally substituted for $(x, y)$ in the functional equation.
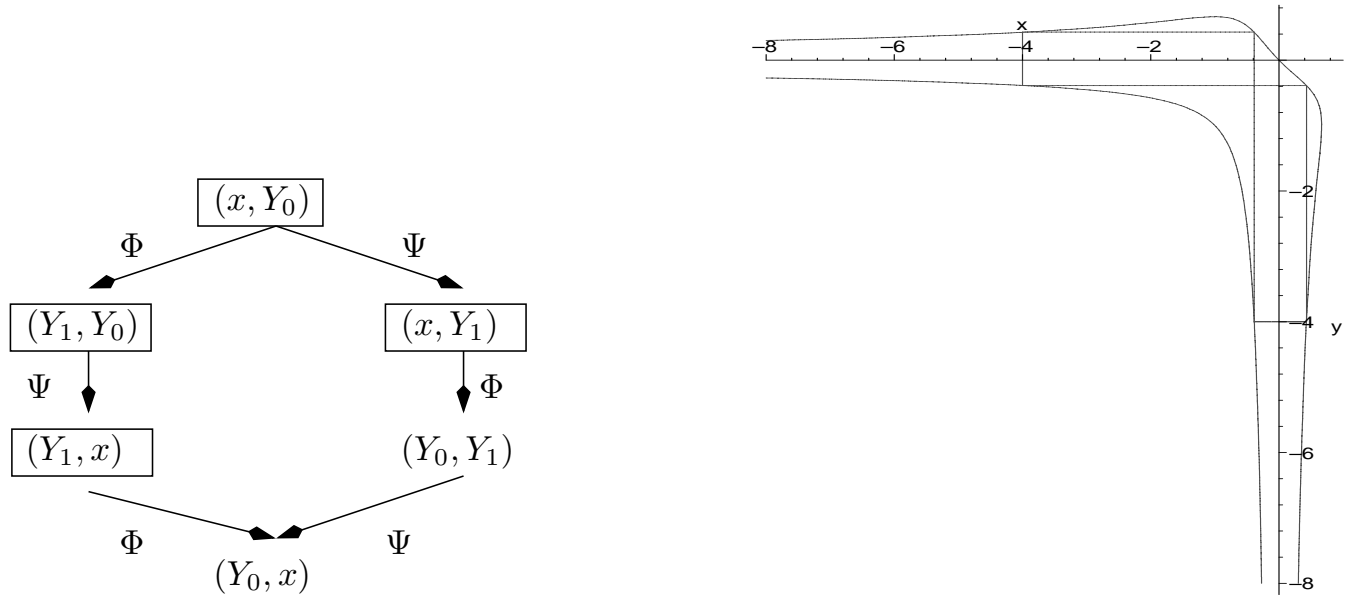

Figure 6: The diagram of roots for 1234-avoiding permutations, and its geometric counterpart (for $t=0.4$ ).

We thus obtain four equations relating the series $R$ and $S$ :

$$
\begin{cases}t\left(1+Y_{0}\right)\left(1-x Y_{0}\right) R(\bar{x}) & +t(1+x)^{2} S\left(Y_{0}\right)=Y_{0}(1+x)\left(1-x Y_{0}\right), \\ t\left(1+Y_{1}\right)\left(1-x Y_{1}\right) R(\bar{x}) & +t(1+x)^{2} S\left(Y_{1}\right)=Y_{1}(1+x)\left(1-x Y_{1}\right), \\ t\left(1+Y_{0}\right)\left(1-Y_{1} Y_{0}\right) R\left(\bar{Y}_{1}\right) & +t\left(1+Y_{1}\right)^{2} S\left(Y_{0}\right)=Y_{0}\left(1+Y_{1}\right)\left(1-Y_{1} Y_{0}\right), \\ t(1+x)\left(1-Y_{1} x\right) R\left(\bar{Y}_{1}\right) & +t\left(1+Y_{1}\right)^{2} S(x)=x\left(1+Y_{1}\right)\left(1-Y_{1} x\right) .\end{cases}
$$

By eliminating $R(\bar{x})$, we obtain a relation between $S\left(Y_{0}\right)$ and $S\left(Y_{1}\right)$, which is symmetric in $Y_{0}$ and $Y_{1}$ :

$$
\frac{\left(1+Y_{1}\right)\left(1-x Y_{1}\right) S\left(Y_{0}\right)-\left(1+Y_{0}\right)\left(1-x Y_{0}\right) S\left(Y_{1}\right)}{Y_{0}-Y_{1}}=\frac{\left(1-x Y_{1}\right)\left(1-x Y_{0}\right)}{t(1+x)}=\frac{1+\bar{x}}{1-t} .
$$

Let us denote

$$
S(y)=y G(1,1+y)=\sum_{i \geq 1} S_{i} y^{i}
$$


where $S_{i}$ is a series in $t$. Both sides of (14) are power series in $t$ with polynomial coefficients in $\bar{x}$. Extracting the constant term (in $\bar{x}$ ) gives

$$
S_{2}=\frac{S_{1}-1}{t}
$$

By eliminating $R\left(\bar{Y}_{1}\right)$ from (13), we obtain an equation that is similar to (14), with $x$ and $Y_{1}$ exchanged. It involves $S(x)$ and $S\left(Y_{0}\right)$. We combine it with (14) so as to form another symmetric expression in $Y_{0}$ and $Y_{1}$, now based on a sum:

$$
\begin{aligned}
2 t\left(1+Y_{0}\right)\left(1+Y_{1}\right)(1 & \left.-Y_{0} Y_{1}\right) S(x)-t(x+1)\left(\left(1+Y_{1}\right)\left(1-x Y_{1}\right) S\left(Y_{0}\right)+\left(1+Y_{0}\right)\left(1-x Y_{0}\right) S\left(Y_{1}\right)\right) \\
& =\left(1-x Y_{1}\right)\left(2 x-Y_{0}-Y_{1}-Y_{0} Y_{1} x-x Y_{0}^{2}+2 Y_{0}^{2} Y_{1}\right) .
\end{aligned}
$$

Let us denote the right-hand side of (16) by $C\left(Y_{0}, Y_{1}\right)$. One can separate the symmetric and anti-symmetric parts of $C$ (with respect to $Y_{0}$ and $Y_{1}$ ) by writing

$$
\begin{aligned}
C\left(Y_{0}, Y_{1}\right)= & \frac{1}{2}\left(C\left(Y_{0}, Y_{1}\right)+C\left(Y_{1}, Y_{0}\right)\right)+\frac{1}{2}\left(C\left(Y_{0}, Y_{1}\right)-C\left(Y_{1}, Y_{0}\right)\right) \\
= & \frac{(1+\bar{x})(1-t-t \bar{x})(1-t x-x-2 t-t \bar{x})}{(1-t)^{2}}+ \\
& \frac{\left(Y_{0}-Y_{1}\right)(1+x)(1+t x-x-t-2 t \bar{x})}{1-t} .
\end{aligned}
$$

We also express the coefficient of $S(x)$ as a rational function of $t$ and $x$, and thus obtain

$$
\begin{array}{r}
\frac{t \bar{x}(1-t)^{2}}{2(1-t-t \bar{x})^{2}}\left(\left(1+Y_{0}\right)\left(1-x Y_{0}\right) S\left(Y_{1}\right)+\left(1+Y_{1}\right)\left(1-x Y_{1}\right) S\left(Y_{0}\right)\right)-\frac{\bar{x}^{2}(1-t x-x-2 t-t \bar{x})}{2(1-t-t \bar{x})} \\
=\bar{x}^{2} t S(x)-A(x)
\end{array}
$$

where

$$
A(x)=\frac{\bar{x}\left(Y_{1}-Y_{0}\right)(1-t)(1-x+t x-t-2 t \bar{x})}{2(1-t-t \bar{x})^{2}} .
$$

Given the value of $Y_{0}$ and $Y_{1}$, this is exactly the series $A(x)$ defined in Theorem 12. By Lemma 13, the left-hand side of the above identity is a series in $t$ with coefficients in $\mathbb{Q}[\bar{x}]$ (and, actually, in $\bar{x} \mathbb{Q}[\bar{x}]$ ). Extracting the nonnegative part gives

$$
t \bar{x}^{2} S(x)-t \bar{x} S_{1}=A^{\geq}(x) .
$$

Extracting the constant term in $x$ yields

$$
t S_{2}=\left[x^{0}\right] A(x) .
$$

In view of (15), we finally obtain

$$
S_{1}=1+\left[x^{0}\right] A(x) .
$$

Note that $S_{1}$, the coefficient of $y$ in $S(y)=y G(1,1+y)$, is exactly $G(1,1)$, the length generating function of 1234-avoiding permutations. The expression of $S(x)=x G(1,1+x)$ is then obtained from (18), and this concludes the proof of Theorem 12. 
Remark. Let us explain where the change of variables $w=1+\bar{x}, v=1+y$ comes from. Recall that we start from (10), and that the replacement of $u$ by $w / v$ is natural in view of the right-hand side. The kernel is thus

$$
(v-1)(v-w)-t\left(v^{2}+w^{2}-v w-v w^{2}\right) .
$$

As a polynomial in $v$, it has two roots $V_{0}$ and $V_{1}$, with $V_{0}=w+O(t)$ and $V_{1}=1+O(t)$. We obtain the diagram of Figure 7 . The pairs that can be substituted for $(w, v)$ in the equation are framed.

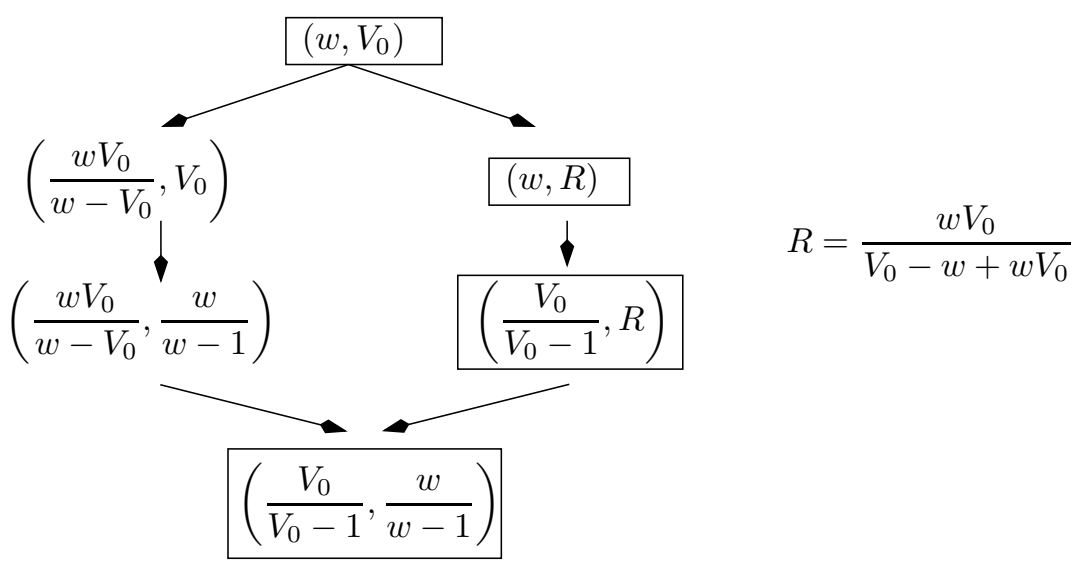

Figure 7: The orbit of $\left(w, V_{0}\right)$.

We thus obtain four equations, involving $G(w, 1), G(V /(V-1), 1)$, and $G(1, V)$, $G(1, R), G(1, w /(w-1))$ (for the sake of simplicity, we denote $V_{0}$ by $V$ ). We can eliminate $G(w, 1)$ and $G(V /(V-1), 1)$ : this gives two equations between three specializations of $G(1, v)$, namely $G(1, V), G(1, R), G(1, w /(w-1))$. We observe that $V$ and $R$ are algebraic functions of $w$, with

$$
V+R=\frac{(1+w)(1-t w)}{1-t} \quad \text { and } \quad V R=\frac{w(1-t w)}{1-t} .
$$

We have learned in $[7,8]$ that it would be nice to see the symmetric functions of $V$ and $R$ as polynomials in a variable $\bar{x}$ (and rational functions of $t$ ) while $G(1, w /(w-1)$ ) would be a power series in $t$ with nonnegative powers in $x$. These two conditions are met by setting $w=1+\bar{x}$. Then $G(w /(w-1))$ becomes $G(1,1+x)$, and, given that the equations involve $G(1, V)$, it only makes sense to write $V=1+Y$, that is, to set $v=1+y$ in the original equation.

Proof of Theorem 9. Let $Z(t ; x) \equiv Z$ be the unique power series in $t$, with constant term zero, such that

$$
Z=t(1+\bar{x})\left(\frac{x}{1-Z}+Z\right) .
$$


Then the series $A$ of Theorem 12 is

$$
A=\frac{(1-2 Z)(x-Z)(1-x-Z)}{2 x^{3}} .
$$

The coefficient of $t^{0}$ in $A$ is $\bar{x}^{2}(1-x) / 2$, and for $n \geq 1$, the Lagrange inversion formula gives

$$
\left[t^{n}\right] A=\frac{1}{2 n x^{3}}\left[t^{n-1}\right]\left((1+\bar{x})^{n}\left(\frac{x}{1-t}+t\right)^{n}\left(-6 t^{2}+6 t+2 x^{2}-2 x-1\right)\right) .
$$

Writing

$$
\left(\frac{x}{1-t}+t\right)^{n}=\sum_{k}\left(\begin{array}{l}
n \\
k
\end{array}\right) \frac{x^{k}}{(1-t)^{k}} t^{n-k} \quad \text { and } \quad \frac{1}{(1-t)^{k}}=\sum_{\ell}\left(\begin{array}{c}
k+\ell-1 \\
k-1
\end{array}\right) t^{\ell},
$$

we obtain

$$
\begin{aligned}
{\left[t^{n}\right] A } & =\frac{(1+\bar{x})^{n}}{2 n x^{3}} \sum_{k \geq 1} x^{k}\left(\begin{array}{l}
n \\
k
\end{array}\right)\left(-6\left(\begin{array}{c}
2 k-4 \\
k-1
\end{array}\right)+6\left(\begin{array}{c}
2 k-3 \\
k-1
\end{array}\right)+\left(2 x^{2}-2 x-1\right)\left(\begin{array}{c}
2 k-2 \\
k-1
\end{array}\right)\right) \\
& =\frac{(1+\bar{x})^{n}}{n x^{3}} \sum_{k \geq 1} x^{k}\left(\begin{array}{l}
n \\
k
\end{array}\right)\left(\begin{array}{c}
2 k-2 \\
k-1
\end{array}\right)\left(\frac{k}{2(2 k-3)}+x^{2}-x\right) .
\end{aligned}
$$

By Theorem 12, the coefficient of $x^{0}$ in this Laurent polynomial is the number of 1234avoiding permutations of length $n$. The extraction of this coefficient gives

$$
\begin{aligned}
{\left[x^{0} t^{n}\right] A=} & \frac{1}{n} \sum_{k \geq 1}\left(\begin{array}{l}
n \\
k
\end{array}\right)\left(\begin{array}{c}
2 k-2 \\
k-1
\end{array}\right)\left(\begin{array}{c}
n \\
k-3
\end{array}\right) \frac{k}{2(2 k-3)}+ \\
& \frac{1}{n} \sum_{k \geq 1}\left(\begin{array}{l}
n \\
k
\end{array}\right)\left(\begin{array}{c}
2 k-2 \\
k-1
\end{array}\right)\left(\left(\begin{array}{c}
n \\
k-1
\end{array}\right)-\left(\begin{array}{c}
n \\
k-2
\end{array}\right)\right) .
\end{aligned}
$$

Summing the $(k+1)$ th term in the first sum with the $k$ th term of the second sum gives the desired expression.

\section{Vexillary involutions}

We now focus on involutions avoiding the pattern 2143. These involutions are also called vexillary. Vexillary permutations first appeared in relation with the geometry of flag varieties and Schubert polynomials [25]. Our aim is to recover and refine the following result.

Theorem 14 The number of vexillary involutions of $\mathcal{S}_{n}$ is the nth Motzkin number:

$$
\sum_{k=0}^{\lfloor n / 2\rfloor} \frac{1}{k+1}\left(\begin{array}{c}
2 k \\
k
\end{array}\right)\left(\begin{array}{c}
n \\
2 k
\end{array}\right) \text {. }
$$



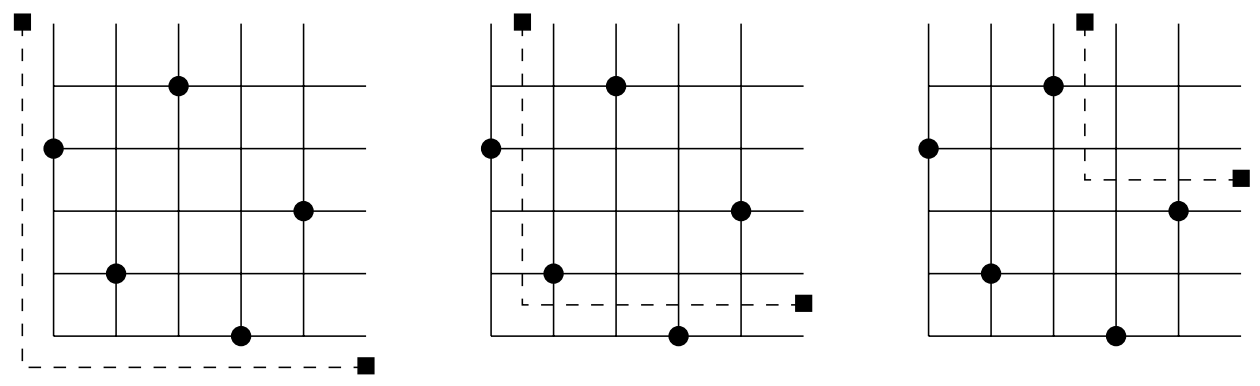

Figure 8: The involution $(1,4)(2)(3,5)=42513$ avoids 2143 . Exactly 3 involutions of length 7 can be obtained by inserting a 2-cycle in $\sigma$. These cycles are $(1,7),(2,7)$ and $(4,7)$.

This is sequence A001006 in the on-line Encyclopedia of Integer Sequences [31]. It starts with $1,2,4,9,21,51,127 \ldots$ The above result was first conjectured by Guibert [19] in 1995, and turned out to be unexpectedly hard to prove. It was finally proved in a complicated bijective manner by Guibert, Pergola and Pinzani [21] in 2001. Note that a related conjecture, which also dates back to 1995, has been proved very recently: the number of 1432-avoiding involutions of length $n$ is also given by the $n$th Motzkin number $[19,22]$.

\subsection{Recursive construction of 2143-avoiding involutions}

Let $\sigma$ be a vexillary involution of length $n$ and let $i=\sigma(n)$. The involution obtained by deleting the cycle containing $n$ and then replacing each letter $j>i$ by $j-1$ also avoids 2143. Conversely, let us try to construct a vexillary involution by adding a new cycle to $\sigma$. Clearly, adding $(n+1)$ as a new fixed point leaves the involution vexillary. Now, let us try to insert a cycle $(i, n+2)$, with $1 \leq i \leq n+1$. That is to say, in the word representation of $\sigma$, we insert $(n+2)$ in the $i$ th position, then we add 1 to all letters $j \geq i$, and finally write the letter $i$ at the end of the word. Alternatively, Figure 8 describes the construction on the graph of the involution. We say that $i$ is an active site if the insertion of the cycle $(i, n+2)$ gives a vexillary involution. Clearly, all sites located to the left of the first descent of $\sigma$ are active. So are certain sites located to the right of the first descent, but these active sites are not necessarily consecutive (Figure 8). Let us introduce the two following parameters: the position $p$ of the first descent,

$$
p= \begin{cases}\min \{k \geq 2: \sigma(k-1)>\sigma(k)\} & \text { if } \sigma \text { contains } 21 \\ n+1 & \text { otherwise }\end{cases}
$$

and the number $q$ of active sites. Note that $p \leq q$. The parameters of the empty involution are $(1,1)$. A careful examination gives the following lemma.

Lemma $15([\mathbf{1 9}, \mathbf{2 1}])$ Let $\sigma$ be a vexillary involution of length $n$, different from $12 \cdots n$. Let $(p, q)$ denote its parameters. The insertion of a new cycle in $\sigma$ gives

- one vexillary involution of length $n+1$, of parameters $(p, p)$, 
- $q$ vexillary involutions of length $n+2$, of parameters

$$
\begin{aligned}
& (2, q+1),(3, q+1), \ldots,(p+1, q+1), \\
& (p, q),(p, q-1), \ldots,(p, p+1) \quad \text { for } p<q .
\end{aligned}
$$

The insertion of a new cycle in the involution $12 \cdots n$, which has parameters $(p, q)=$ $(n+1, n+1)$, gives

- one vexillary involution of length $n+1$, of parameters $(p+1, p+1)$,

- $q$ vexillary involutions of length $n+2$, of parameters

$$
(2, q+1),(3, q+1), \ldots,(p+1, q+1) .
$$

Note that the only difference between the identity and a generic involution lies in the parameters of the involution obtained by adding a fixed point.

Corollary 16 Let $G(t ; u, v) \equiv G(u, v)$ denote the generating function of 2143-avoiding involutions, counted by their length (variable $t$ ) and parameters (variables $u$ and $v$ ). Then

$$
\left(1+\frac{t^{2} u^{2} v}{1-u}+\frac{t^{2} v}{1-v}\right) G(u, v)=\frac{u v(1-t)}{1-u v t}+t\left(1+\frac{t v}{1-v}\right) G(u v, 1)+\frac{t^{2} u^{2} v}{1-u} G(1, v) .
$$

Remark. The kernel of the above equation is the same (up to the substitution of $t$ by $t^{2}$ ) as the kernel of the equation satisfied by the generating function of 1234-avoiding permutations (or of vexillary permutations; see the remark that follows Corollary 11). This is closely related to the fact that vexillary involutions of length $2 n$ having no fixed points are in bijection with vexillary permutations of length $n$ [21, p. 156]. We explain at the end of this section how to interpolate between vexillary permutations and vexillary involutions.

\subsection{Solution of the functional equation for vexillary involutions}

Theorem 17 Let $Z(t) \equiv Z$ be the unique power series in $t$ satisfying

$$
Z=t\left(1+Z+Z^{2}\right)
$$

The generating function $G(t ; u, v)$ that counts vexillary involutions by the length, the position of the first descent and the number of active sites is algebraic of degree 2:

$$
G(t ; u, v)=\frac{u v\left(1-t v(1+Z)+t^{2} u v^{2} Z\right)}{(1-t u v)(1-t u v Z)(1-t v(1+Z))} .
$$

In particular, $G(t ; 1,1)=Z / t$, and the Lagrange inversion formula gives Theorem 14 .

Proof. As can be expected from the similarities between the equations of Corollaries 11 and 16, we are going to follow exactly the same steps as in the solution of the functional equation for 1234-avoiding permutations. The reason why the generating function of 
vexillary involutions is algebraic (hence simpler) is that in the counterpart of (17), the asymmetric part will be zero.

Let us simply sketch the first few steps. Again, we set $u=(1+\bar{x}) /(1+y)$ and $v=1+y$ in the equation of Corollary 16. The equation we thus obtain has the same kernel as (11) (up to the replacement of $t$ by $t^{2}$ ). In particular, the roots of the kernel are given by (12) (with $t$ replaced by $t^{2}$ ), and the diagram of the roots is that of Figure 6 . The pairs $(X, Y)$ that can be substituted for $(x, y)$ in the kernel give four equations relating the series $R$ and $S$, from which we form two equations involving only $S$, in which $S\left(Y_{0}\right)$ and $S\left(Y_{1}\right)$ play symmetric roles:

$$
\frac{\left(1-x Y_{1}\right)\left(t-Y_{1}(1-t)\right) S\left(Y_{0}\right)-\left(1-x Y_{0}\right)\left(t-Y_{0}(1-t)\right) S\left(Y_{1}\right)}{Y_{0}-Y_{1}}=\frac{t(1+\bar{x})}{(1+t)(1-t-t \bar{x})}
$$

and

$$
\begin{gathered}
\frac{(x-t x-t)\left(1-t^{2}\right)(1+t)}{1-t^{2}(1+\bar{x})}\left(\left(1-x Y_{1}\right)\left(t-Y_{1}(1-t)\right) S\left(Y_{0}\right)+\left(1-x Y_{0}\right)\left(t-Y_{0}(1-t)\right) S\left(Y_{1}\right)\right) \\
=2 t \bar{x}\left(1-t-t x-2 t^{2}-t^{2} x-t^{2} \bar{x}\right) S(x)+t(1+\bar{x})(1-2 x) .
\end{gathered}
$$

The left-hand side of (19) is a series in $t$ with polynomial coefficients in $\bar{x}$. Again, let us denote

$$
S(y)=y G(1,1+y)=\sum_{i \geq 1} S_{i} y^{i}
$$

where $S_{i}$ is a series in $t$. Extracting the constant term (in $\bar{x}$ ) of (19) gives

$$
S_{2}=\frac{S_{1}(1-t)-1}{t^{2}}
$$

Now, extracting the positive part of (20) gives

$$
\bar{x}\left(1-t-t x-2 t^{2}-t^{2} x-t^{2} \bar{x}\right) S(x)-\left(1-t-2 t^{2}-t^{2} x-t^{2} \bar{x}\right) S_{1}+t^{2} S_{2}-x=0 .
$$

Using the relation (21) between $S_{1}$ and $S_{2}$, we finally obtain:

$$
t^{2} x(1+x)^{2} S_{1}+\left(x-t x-t x^{2}-t^{2} x^{2}-2 t^{2} x-t^{2}\right) S(x)=x^{2}(1+x) .
$$

The coefficient of $S(x)$ in this equation is a quadratic polynomial in $x$. One of its roots is a power series in $t$,

$$
X=\frac{1-t-2 t^{2}-\sqrt{(1+t)(1-3 t)}}{2 t(1+t)} .
$$

Applying the kernel method to (22) finally yields

$$
S_{1}=\frac{X}{t^{2}(1+X)}=\frac{1-t-\sqrt{(1+t)(1-3 t)}}{2 t^{2}} .
$$


From now on, it is convenient to express $t$ in terms of the series $Z$ defined in the theorem. One first obtains the expression of $S(x)=x G(1,1+x)$ thanks to (22). The counterpart of System (13) (which contains four equations and five unknown series) provides the expression of $R(\bar{x})=x G(1+\bar{x}, 1)$, and finally $G(u, v)$ is derived using the equation of Corollary 16. We omit the details.

Remark: from vexillary involutions to vexillary permutations. As recalled right after Corollary 16, vexillary involutions of length $2 n$ having no fixed point are in bijection with vexillary permutations of length $n$. Hence, it seems natural to count vexillary involutions by their length and number of fixed points, in order to obtain a result that encompasses the enumeration of vexillary permutations and involutions. It is very easy to enrich the functional equation of Corollary 16 so as to take into account the number of fixed points: indeed, in the recursive construction of vexillary involutions, a new fixed point is created each time one inserts a cycle of length 1 . This gives:

$$
\left(1+\frac{t^{2} u^{2} v}{1-u}+\frac{t^{2} v}{1-v}\right) G(u, v)=\frac{u v(1-z t)}{1-u v t z}+t\left(z+\frac{t v}{1-v}\right) G(u v, 1)+\frac{t^{2} u^{2} v}{1-u} G(1, v),
$$

where $G(u, v) \equiv G(t, z ; u, v)$ now counts vexillary involutions by their length $(t)$, number of fixed points $(z)$, and parameters $(u$ and $v)$. Our approach still applies; as one can expect, the details of the calculations involve a mixture of Sections 3 and 4 . The generating function $G(t, z ; 1,1)$ is given by

$$
((1-t z)(z-t)-\sqrt{\Delta(t, z)}) G(t, z ; 1,1)=\left[x^{0}\right]\left(\left(Y_{0}-Y_{1}\right) A(t, z)\right)+B(t, z)
$$

where $Y_{0}$ and $Y_{1}$ are again the roots of the kernel,

$$
\Delta(t, z)=(1-z t)(z-t)\left(z-t-t z^{2}-3 t^{2} z\right)
$$

and $A(t, z)$ and $B(t, z)$ are two explicit algebraic series lying in $\mathbb{Q}(t, z, \sqrt{\Delta(t, z)})$.

\section{$5 \quad$ Involutions avoiding 54321}

We finally focus on involutions containing no decreasing subsequence of length 5 . Our aim is to recover and refine the following result.

Theorem 18 The number of 54321-avoiding involutions of $\mathcal{S}_{n}$ is

$$
C_{\lceil n / 2\rceil} C_{1+\lfloor n / 2\rfloor}
$$

where $C_{n}=\left(\begin{array}{c}2 n \\ n\end{array}\right) /(n+1)$ denotes the nth Catalan number.

This is sequence AA005817 in the on-line Encyclopedia of Integer Sequences [31]. It starts with $1,2,4,10,25,70 \ldots$ By the Robinson-Schensted correspondence, these numbers also count standard Young tableaux of height at most 4. The above result was first obtained via a bijective proof by Gouyou-Beauchamps [18], and then by Gessel [16], using symmetric functions. See also exercise 7.16 in [35]. 


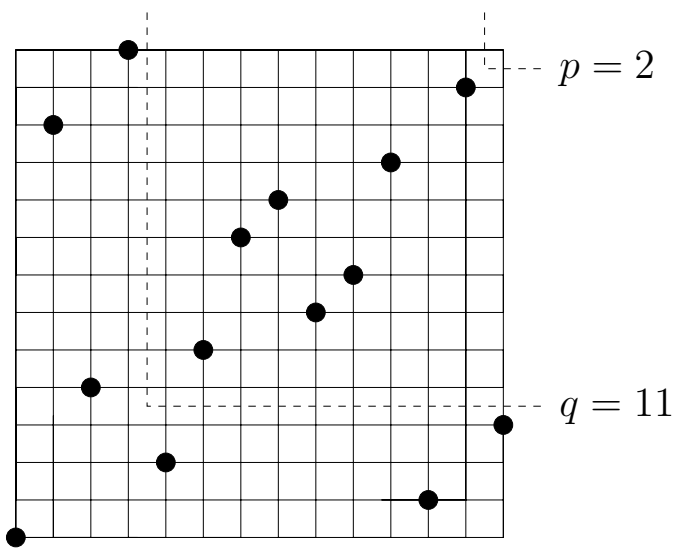

Figure 9: A 54321-avoiding involution $\sigma$ of length 14 and parameters $(2,11)$. Exactly 11 involutions of length 16 can be obtained by inserting a 2-cycle in $\sigma$. These cycles are $(5,16),(6,16), \ldots,(15,16)$.

\subsection{Recursive construction of 54321-avoiding involutions}

Let $\sigma$ be a 54321-avoiding involution of length $n$. Assume $\sigma(n)=i$. The involution obtained by deleting the cycle containing $n$ and then replacing each letter $j>i$ by $j-1$ also avoids 54321. Conversely, let us try to construct a 54321-avoiding involution by adding a new cycle to $\sigma$. Clearly, adding $(n+1)$ as a new fixed point leaves the involution 54321 -avoiding. Now, let us try to insert a cycle $(i, n+2)$, with $1 \leq i \leq n+1$, as described in the previous section. As we want the new involution to avoid 54321, this intuitively means that $i$ should not be "too small". A careful examination leads us to introduce two additional parameters.

For $k \geq 0$, let us define the restriction of $\sigma$ to its $k$ largest elements as the involution obtained by retaining only the cycles whose elements are among the $k$ largest. In the graph of the involution, the restriction is the set of points lying in the North-East square corner of size $k$. For instance, the restriction of $\sigma=(1)(2,12)(3,5)(4,14)(6)(7,9)(8,10)(11)(13)$ (a 54321-avoiding involution) to its 4 largest elements is (11)(13). Let $p \in[1, n+1]$ be the largest $j$ such that the restriction of $\sigma$ to its $j-1$ largest elements is empty. Let $q \in[1, n+1]$ be the largest $j$ such that the restriction of $\sigma$ to its $j-1$ largest elements is 321-avoiding. Clearly, $p \leq q$. The parameters of the empty involution are $(1,1)$. The parameters of the above involution $\sigma$ are $(2,11)$ (Figure 9).

Lemma 19 Let $\sigma$ be a 54321-avoiding involution of length $n$. The insertion of a new cycle in $\sigma$ gives

- one 54321-avoiding involution of length $n+1$, of parameters $(1, q+1)$,

- $q$ 54321-avoiding involutions of length $n+2$, of parameters

$$
\begin{aligned}
& (2, q+2),(3, q+2), \ldots,(p+1, q+2), \\
& (p+1, p+2),(p+1, p+3), \ldots,(p+1, q+1) \quad \text { for } p<q .
\end{aligned}
$$


The proof is left to the reader.

Corollary 20 Let $G(t ; u, v) \equiv G(u, v)$ denote the generating function of 54321-avoiding involutions, counted by their length (variablet) and parameters (variables $u$ and $v$ ). Then

$$
\left(1+\frac{t^{2} u^{2} v^{2}}{1-u}+\frac{t^{2} u v^{2}}{1-v}\right) G(u, v)=u v+\frac{t^{2} u v^{2}}{1-v} G(u v, 1)+t u v\left(1+\frac{t u v}{1-u}\right) G(1, v) .
$$

\subsection{Solution of the functional equation for 54321-avoiding invo- lutions}

Theorem 21 Let $Y(t ; x) \equiv Y$ be the unique formal power series in $t$ such that

$$
Y=t^{2}(1+x)(1+\bar{x})(1+Y)^{2} .
$$

This series has coefficients in $\mathbb{Q}[x, \bar{x}]$, with $\bar{x}=1 / x$. The series $G(t ; u, 1)$ that counts 54321-avoiding involutions by their length and the parameter $p$ defined above satisfies

$$
t G(t ; 1+x, 1)=-1+((1-\bar{x})(1+t(1+x)(1+\bar{x}))(1+Y))^{\geq} .
$$

The Lagrange inversion formula gives:

Corollary 22 The series $G(t ; u, 1)$ admits the following expansions:

$$
G(t ; 1+x, 1)=\sum_{n \geq 0} t^{n} \sum_{i=0}^{1+\lfloor n / 2\rfloor} \frac{x^{i}(2 i+1) C_{\lceil n / 2\rceil}}{2\lfloor n / 2\rfloor+3}\left(\begin{array}{c}
2\lfloor n / 2\rfloor+3 \\
\lfloor n / 2\rfloor-i+1
\end{array}\right),
$$

and

$$
G(t ; u, 1)=\sum_{n \geq 0} t^{n} C_{\lceil n / 2\rceil} \sum_{i=0}^{1+\lfloor n / 2\rfloor} \frac{i u^{i}}{\lfloor n / 2\rfloor+1}\left(\begin{array}{c}
2\lfloor n / 2\rfloor-i+1 \\
\lfloor n / 2\rfloor
\end{array}\right) .
$$

Note that the case $x=0$ of $(23)$ is Theorem 18 .

Proof of Theorem 21. Let us perform the following change of variables in the equation of Corollary 20:

$$
u=\frac{w}{v}, \quad w=1+y, \quad v=1+\frac{y}{1+x+x y}=\frac{(1+x)(1+y)}{1+x+x y} .
$$

This will greatly simplify the diagram of the roots of the kernel. The functional equation becomes

$$
\begin{aligned}
& \frac{x y-t^{2}(1+x)^{2}(1+y)^{2}}{1+y} G\left(1+\frac{x y}{1+x}, 1+\frac{y}{1+x+x y}\right) \\
& \quad=x y-t^{2} x(1+x)(1+y) G(1+y, 1)+t(x y-t(1+x)(1+y)) G\left(1,1+\frac{y}{1+x+x y}\right) .
\end{aligned}
$$


Note that the kernel is now symmetric in $x$ and $y$. As a polynomial in $y$, it admits two roots:

$$
\begin{aligned}
Y_{0}(x) & =\frac{1-\sqrt{1-4 t^{2}(1+x)(1+\bar{x})}}{2 t^{2}(1+x)(1+\bar{x})}-1 \\
& =(1+x)(1+\bar{x}) t^{2}+2(1+x)^{2}(1+\bar{x})^{2} t^{4}+O\left(t^{6}\right), \\
Y_{1}(x) & =\frac{1+\sqrt{1-4 t^{2}(1+x)(1+\bar{x})}}{2 t^{2}(1+x)(1+\bar{x})}-1 \\
& =\frac{1}{(1+x)(1+\bar{x}) t^{2}}-2-(1+x)(1+\bar{x}) t^{2}+O\left(t^{4}\right) .
\end{aligned}
$$

Observe that $Y_{0} Y_{1}=1$, and that the series $Y_{i}$ are symmetric in $x$ and $\bar{x}$. The diagram of the pairs $(X, Y)$ that cancel the kernel, obtained by starting from the pair $\left(x, Y_{0}\right)$, is shown on the left of Figure 10. However, given the symmetry in $x$ and $y$ of the kernel, the pairs shown on the right-hand side of this figure also cancel the kernel. A geometric illustration of these diagrams is given in Figure 11.
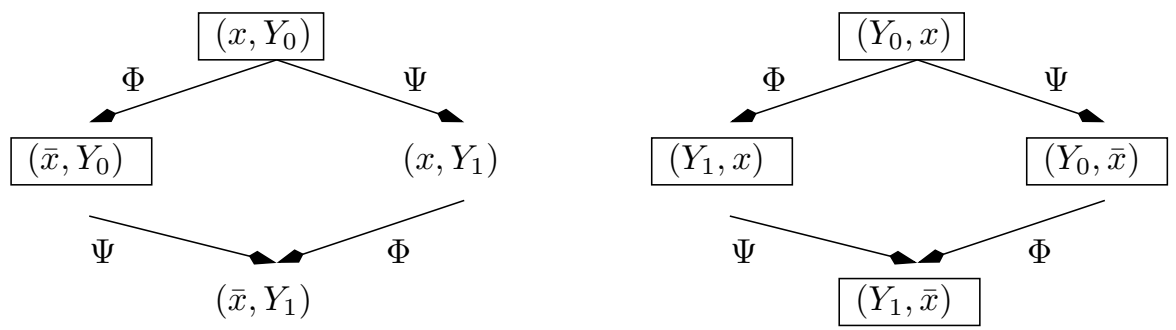

Figure 10: The diagram of roots for 54321-avoiding involutions.

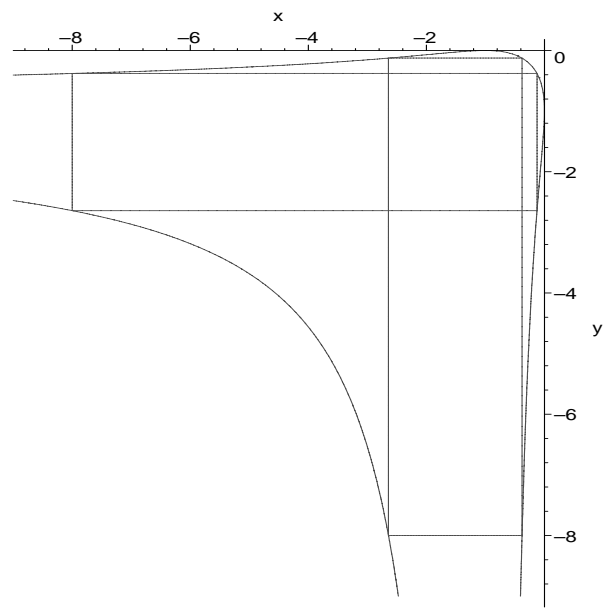

Figure 11: The real part of the curve $K(t ; x, y)=0$ for $t=0.4$. 
Exactly 6 pairs in these diagrams can be substituted for $(x, y)$ in the functional equation. In particular, the pairs $\left(x, Y_{0}\right),\left(\bar{x}, Y_{0}\right),\left(Y_{1}, x\right)=\left(1 / Y_{0}, x\right)$ and $\left(Y_{1}, \bar{x}\right)=\left(1 / Y_{0}, \bar{x}\right)$ give the following system:

$$
\left\{\begin{array}{lll}
x t^{2}(1+x)\left(1+Y_{0}\right) G\left(1+Y_{0}, 1\right) & +t\left(t(1+x)\left(1+Y_{0}\right)-x Y_{0}\right) G\left(1, \frac{(1+x)\left(1+Y_{0}\right)}{1+x+x Y_{0}}\right)=x Y_{0}, \\
t^{2}(1+x)\left(1+Y_{0}\right) G\left(1+Y_{0}, 1\right) & +t x\left(t(1+x)\left(1+Y_{0}\right)-Y_{0}\right) G\left(1, \frac{(1+x)\left(1+Y_{0}\right)}{1+x+Y_{0}}\right)=x Y_{0}, \\
t^{2}(1+x)\left(1+Y_{0}\right) G(1+x, 1)+t Y_{0}\left(t(1+x)\left(1+Y_{0}\right)-x\right) G\left(1, \frac{(1+x)\left(1+Y_{0}\right)}{1+x+Y_{0}}\right)=x Y_{0}, \\
t^{2}(1+x)\left(1+Y_{0}\right) G(1+\bar{x}, 1)+t Y_{0}\left(t(1+x)\left(1+Y_{0}\right)-1\right) G\left(1, \frac{(1+x)\left(1+Y_{0}\right)}{1+x+x Y_{0}}\right)=Y_{0} .
\end{array}\right.
$$

This system of four equations relates five unknown series. Hence, we can derive from it an equation between any two of them. In particular, upon noticing that

$$
\left(t(1+x)\left(1+Y_{0}\right)-x Y_{0}\right)\left(t(1+x)\left(1+Y_{0}\right)-x\right)=x\left(t(1+x)\left(1+Y_{0}\right)-Y_{0}\right)\left(t(1+x)\left(1+Y_{0}\right)-1\right),
$$

the equation that links $G(1+x, 1)$ and $G(1+\bar{x}, 1)$ can be written

$$
t \bar{x} G(1+\bar{x}, 1)-t G(1+x, 1)=\bar{x}^{2}(1-x)\left(t(1+x)^{2}\left(1+Y_{0}\right)+x Y_{0}\right) .
$$

What remains is to extract the nonnegative part in $x$ to obtain the announced result. A straightforward application of the Lagrange inversion formula gives the first expansion of Corollary 22. For the second one, we use Lemma 6. We omit the details.

Acknowledgements. I am very grateful to Frédéric Chyzak and Olivier Guibert for interesting discussions on the topic of this paper. I also thank Einar Steingrímsson for his comments which helped to improve the manuscript.

\section{References}

[1] E. Babson and E. Steingrímsson. Generalized permutation patterns and a classification of the Mahonian statistics. Sém. Lothar. Combin., 44:Art. B44b, 18 pp. (electronic), 2000.

[2] C. Banderier, M. Bousquet-Mélou, A. Denise, P. Flajolet, D. Gardy, and D. GouyouBeauchamps. Generating functions for generating trees. Discrete Math., 246(1-3):2955, 2002. Formal Power Series and Algebraic Combinatorics (Barcelona, 1999).

[3] C. Banderier and P. Flajolet. Basic analytic combinatorics of directed lattice paths. Theoret. Comput. Sci., 281(1-2):37-80, 2002.

[4] R. J. Baxter. Dichromatic polynomials and Potts models summed over rooted maps. Ann. Comb., 5(1):17-36, 2001. 
[5] M. Bóna. Exact enumeration of 1342-avoiding permutations: a close link with labeled trees and planar maps. J. Combin. Theory Ser. A, 80(2):257-272, 1997.

[6] M. Bousquet-Mélou. Three osculating walkers. In preparation.

[7] M. Bousquet-Mélou. Walks in the quarter plane: Kreweras' algebraic model. In preparation.

[8] M. Bousquet-Mélou. Counting walks in the quarter plane. In Mathematics and computer science, II (Versailles, 2002), Trends Math., pages 49-67. Birkhäuser, Basel, 2002.

[9] M. Bousquet-Mélou and M. Petkovšek. Linear recurrences with constant coefficients: the multivariate case. Discrete Math., 225(1-3):51-75, 2000. Formal Power Series and Algebraic Combinatorics (Toronto, ON, 1998).

[10] M. Bousquet-Mélou and M. Petkovšek. Walks confined in a quadrant are not always D-finite. Theoret. Comput. Sci., 307:257-276, 2003.

[11] F. R. K. Chung, R. L. Graham, V. E. Hoggatt, Jr., and M. Kleiman. The number of Baxter permutations. J. Combin. Theory Ser. A, 24(3):382-394, 1978.

[12] A. Claesson and T. Mansour. Counting occurrences of a pattern of type $(1,2)$ or $(2,1)$ in permutations. Adv. in Appl. Math., 29(2):293-310, 2002.

[13] S. Dulucq and O. Guibert. Stack words, standard tableaux and Baxter permutations. Discrete Math., 157(1-3):91-106, 1996. Formal Power Series and Algebraic Combinatorics (New Brunswick, NJ, 1994).

[14] G. Fayolle, R. Iasnogorodski, and V. Malyshev. Random walks in the quarter-plane: Algebraic methods, boundary value problems and applications, volume 40 of Applications of Mathematics. Springer-Verlag, Berlin, 1999.

[15] I. Gessel, J. Weinstein, and H. S. Wilf. Lattice walks in $\mathbf{Z}^{d}$ and permutations with no long ascending subsequences. Electron. J. Combin., 5(1):Research Paper 2, 11 pp. (electronic), 1998.

[16] I. M. Gessel. Symmetric functions and P-recursiveness. J. Combin. Theory Ser. A, $53(2): 257-285,1990$.

[17] S. Gire. Arbres, permutations à motifs exclus et cartes planaires : quelques problèmes algorithmiques et combinatoires. PhD thesis, LaBRI, Université Bordeaux 1, 1993.

[18] D. Gouyou-Beauchamps. Standard Young tableaux of height 4 and 5. European J. Combin., 10(1):69-82, 1989.

[19] O. Guibert. Combinatoire des permutations à motifs exclus, en liaison avec mots, cartes planaires et tableaux de Young. PhD thesis, LaBRI, Université Bordeaux 1, 1995. 
[20] O. Guibert and E. Pergola. Enumeration of vexillary involutions which are equal to their mirror/complement. Discrete Math., 224(1-3):281-287, 2000.

[21] O. Guibert, E. Pergola, and R. Pinzani. Vexillary involutions are enumerated by Motzkin numbers. Ann. Comb., 5(2):153-174, 2001.

[22] A. D. Jaggard. Prefix exchanging and pattern avoidance for involutions. Electron. J. Combin., 9(2):Research Paper 16, 2003.

[23] C. Krattenthaler. Permutations with restricted patterns and Dyck paths. Adv. in Appl. Math., 27(2-3):510-530, 2001.

[24] D. Kremer. Permutations with forbidden subsequences and a generalized Schröder number. Discrete Math., 218(1-3):121-130, 2000.

[25] A. Lascoux and M.-P. Schützenberger. Schubert polynomials and the LittlewoodRichardson rule. Lett. Math. Phys., 10(2-3):111-124, 1985.

[26] L. Lipshitz. The diagonal of a $D$-finite power series is D-finite. J. Algebra, 113(2):373$378,1988$.

[27] L. Lipshitz. D-finite power series. J. Algebra, 122:353-373, 1989.

[28] C. L. Mallows. Baxter permutations rise again. J. Combin. Theory Ser. A, 27(3):394396, 1979.

[29] M. Petkovšek, H. S. Wilf, and D. Zeilberger. $A=B$. A K Peters Ltd., Wellesley, MA, 1996.

[30] C. Schensted. Longest increasing and decreasing subsequences. Canad. J. Math., 13:179-191, 1961.

[31] N. J. A. Sloane and S. Plouffe. The encyclopedia of integer sequences. Academic Press Inc., San Diego, CA, 1995. http://www.research.att.com/ njas/sequences/index.html.

[32] Z. Stankova. Classification of forbidden subsequences of length 4. European J. Combin., 17(5):501-517, 1996.

[33] Z. Stankova and J. West. A new class of Wilf-equivalent permutations. J. Algebraic Combin., 15(3):271-290, 2002.

[34] R. P. Stanley. Differentiably finite power series. European J. Combin., 1:175-188, 1980.

[35] R. P. Stanley. Enumerative combinatorics. Vol. 2, volume 62 of Cambridge Studies in Advanced Mathematics. Cambridge University Press, Cambridge, 1999. 
[36] G. Viennot. A bijective proof for the number of Baxter permutations. 3rd Séminaire lotharingien de Combinatoire, Le Klebach, 1981.

[37] J. West. Permutations with forbidden subsequences, and stack-sortable permutations. PhD thesis, MIT, 1990.

[38] J. West. Generating trees and the Catalan and Schröder numbers. Discrete Math., 146(1-3):247-262, 1995.

[39] D. Zeilberger. The umbral transfer-matrix method: I. Foundations. J. Comb. Theory, Ser. A, 91:451-463, 2000. 